\title{
q-DEFORMATIONS OF STATISTICAL MECHANICAL SYSTEMS AND MOTIVES OVER FINITE FIELDS
}

\author{
MATILDE MARCOLLI AND ZHI REN
}

\begin{abstract}
We consider q-deformations of Witt rings, based on geometric operations on zeta functions of motives over finite fields, and we use these deformations to construct $q$-analogs of the Bost-Connes quantum statistical mechanical system. We show that the $q$-deformations obtained in this way can be related to Habiro ring constructions of analytic functions over $\mathbb{F}_{1}$ and to categorifications of Bost-Connes systems.
\end{abstract}

\section{INTRODUCTION}

In the present paper, we study $q$-deformations of the Bost-Connes system, their relation to classical $q$-analog constructions, and the properties of the resulting quantum statistical mechanical systems, in relation to motives over finite fields. In this introductory section we review briefly the main terminology and notation, with reference to the appropriate literature, about the Bost-Connes system, and about some relevant notions of $q$-deformation.

In the rest of the paper, we first consider the $q$-deformations of the Witt $\operatorname{ring} W(A)$ introduced in [20], 21], 22], 23] and we show that, under these deformations $W^{q}(A)$ of the Witt ring, the Bost-Connes algebra remains undeformed. We then introduce some natural modifications of these $q$-deformations, which are motivated by natural geometric operations on zeta functions of schemes of finite type over $\mathbb{F}_{q}$. We show that these modified $q$-deformations give rise to $q$-deformed Bost-Connes algebras. The first geometric deformation we consider corresponds, at the level of zeta functions, to a geometric tower obtained by taking products of a given scheme $X$ with sets of $q^{\ell}$-points. This deformation is similar to the one considered in [20], 21], 22, [23, but it deforms $W_{0}(A)$ leaving the product in the Witt ring $W(A)$ and $\Lambda(A)$ undeformed. The resulting $q$-deformation of the Bost-Connes algebra, in this case, consists of only a mild change that replaces the original integral Bost-Connes algebra $\mathcal{A}_{\mathbb{Z}}$ of [7] with the product $\mathcal{A}_{\mathbb{Z}} \otimes_{\mathbb{Z}} \mathbb{Z}[q]$. We then show that a more interesting $q$-deformation is obtained if, instead of taking products of schemes $X$ with zero-dimensional spaces consisting of $q^{\ell}$-points, one takes products with affine spaces $\mathbb{A}^{\ell}$. Since $\# \mathbb{A}^{\ell}\left(\mathbb{F}_{q}\right)=q^{\ell}$, this deformation generalizes the previous one, in the sense that the previous one occurs as first order term. We then show that, using these deformations one obtains interesting $q$-deformed Bost-Connes algebras that can be related to the constructions of [15], 17], and of [14, and also to the categorifications of Bost-Connes systems of [18. We also discuss the role of $q$-analogs and a $q$-deformation of the Riemann zeta function in this context. Finally, we propose the categorification of Weil numbers and its associated quantum statistical mechanical system constructed in [18] as another possible $q$-deformation of the Bost-Connes algebra that fits into the general framework discussed in this paper. 
1.1. The Bost-Connes quantum statistical mechanical system. In [2, Bost and Connes introduced a quantum statistical mechanical system whose partition function is the Riemann zeta function and whose equilibrium states are related to cyclotomic fields with the Galois action. The algebra of observables of the system is a semigroup crossed product algebra $C^{*}(\mathbb{Q} / \mathbb{Z}) \rtimes \mathbb{N}$, where $C^{*}(\mathbb{Q} / \mathbb{Z})=C(\hat{\mathbb{Z}})$ is generated by abstract roots of unity $e(r)$, $r \in \mathbb{Q} / \mathbb{Z}$, and the elements $n$ in the multiplicative semigroup are realized by isometries $\mu_{n}$. The semigroup $\mathbb{N}$ acts on $C^{*}(\mathbb{Q} / \mathbb{Z})$ by endomorphisms

$$
\rho_{n}(e(r))=\mu_{n} e(r) \mu_{n}^{*}=\frac{1}{n} \sum_{s: n s=r} e(s) .
$$

The time evolution of the system is given by $\sigma_{t}(e(r))=e(r)$ and $\sigma_{t}\left(\mu_{n}\right)=n^{i t} \mu_{n}$, with generator the Hamiltonian $H \epsilon_{k}=\log (k) \epsilon_{k}$, in a representation on $\ell^{2}(\mathbb{N})$. The partition function is the Riemann zeta function,

$$
Z(\beta)=\operatorname{Tr}\left(e^{-\beta H}\right)=\sum_{k \geq 1} k^{-\beta}
$$

The extremal low temperature KMS states are polylogarithm functions evaluated at roots of unity, normalized by the Riemann zeta function, while at zero temperature the KMS states and the symmetry action are related to cyclotomic fields with the Galois action of $\operatorname{Gal}\left(\mathbb{Q}^{a b} / \mathbb{Q}\right)$. We refer the reader to [2] and Chapter 3 of [8] for a detailed discussion of these properties.

The Bost-Connes system was reinterpreted in [6] as a particular example of an "endomotive", a projective system of zero-dimensional varieties with semigroup actions. The analytical properties of the quantum statistical mechanical system and its relation to $L$-functions were also generalized to this context. Moreover, in [7], the Bost-Connes endomotive was related to the geometry of the "field with one element" $\mathbb{F}_{1}$ and its system of extensions (given by roots of unity) as defined by Kapranov-Smirnov [11. In [5], the integral Bost-Connes system considered in [7] was related to Witt rings and the Frobenius and Verschiebung operators. In [18, generalizations and categorifications of the Bost-Connes system were constructed, which include Weil numbers and motives over finite fields.

Further aspects of the relation between the Bost-Connes system and $\mathbb{F}_{1}$-geometry were considered in [15] and [17. Manin proposed in [15] to use the Habiro ring of $q$-functions, [9], as a good notion of analytic geometry and analytic functions over $\mathbb{F}_{1}$, and analogs of the Bost-Connes system based on the Habiro ring were constructed in [17. Relations between the Habiro ring and motives were discussed in [14].

1.2. The integral Bost-Connes algebra. An integral model of the Bost-Connes algebra was introduced in [7, in relation to $\mathbb{F}_{1}$-geometry.

The integral Bost-Connes algebra $\mathcal{A}_{\mathbb{Z}}$ is the algebra generated by the group ring $\mathbb{Z}[\mathbb{Q} / \mathbb{Z}]$ and by elements $\tilde{\mu}_{n}$ and $\mu_{n}^{*}$ satisfying the relations

$$
\begin{gathered}
\tilde{\mu}_{n} x \mu_{n}^{*}=\tilde{\rho}_{n}(x) \\
\mu_{n}^{*} x=\sigma_{n}(x) \mu_{n}^{*} \\
x \tilde{\mu}_{n}=\tilde{\mu}_{n} \sigma_{n}(x),
\end{gathered}
$$

for all $x \in \mathbb{Z}[\mathbb{Q} / \mathbb{Z}]$ and all $n \in \mathbb{N}$, where $\sigma_{n}(x)$ is an endomorphism of $\mathbb{Z}[\mathbb{Q} / \mathbb{Z}]$ given by $\sigma_{n}(e(r))=e(n r)$, while $\tilde{\rho}_{n}(e(r))=\sum_{n r^{\prime}=r} e\left(r^{\prime}\right)$. The elements $\tilde{\mu}_{n}$ and $\mu_{n}^{*}$ also satisfy the 
relations

$$
\begin{gathered}
\tilde{\mu}_{n m}=\tilde{\mu}_{n} \tilde{\mu}_{m}, \quad m, n \in \mathbb{N}, \quad \mu_{n m}^{*}=\mu_{n}^{*} \mu_{m}^{*}, \quad m, n \in \mathbb{N}, \\
\mu_{n}^{*} \tilde{\mu}_{n}=n, \quad n \in \mathbb{N}, \quad \tilde{\mu}_{n} \mu_{n}^{*}=\mu_{n}^{*} \tilde{\mu}_{n}, \quad(n, m)=1 .
\end{gathered}
$$

In [5], the integral BC algebra is related to the universal Witt ring $W_{0}\left(\overline{\mathbb{F}}_{p}\right)$, where $\overline{\mathbb{F}}_{p}$ is the algebraic closure of $\mathbb{F}_{p}$. Moreover, it is shown that that there is a $p$-adic representation of the integral BC algebra into the Big Witt Ring $W\left(\overline{\mathbb{F}}_{p}\right)$, determined by the embedding of $W_{0}\left(\overline{\mathbb{F}}_{p}\right)$ into $W\left(\overline{\mathbb{F}}_{p}\right)$.

1.3. $q$-Deformations. There is a vast literature on the subject of $q$-deformations and $q$ analogs. The basic form of $q$-analog consists of the $q$-integers

$$
[n]_{q}:=\frac{1-q^{n}}{1-q}=1+q+\cdots+q^{n-1} .
$$

Generalizations of the Riemann zeta function based on $q$-integers have been considered, for instance, in [4], [10], [12], and in a different form, suitable for an Euler product expansion, in [26], [27], while $q$-Bernoulli numbers were considered in [3]. A $q$-deformed analog of the polylogarithm function, the $q$-polylogarithm, was considered in [29]. The Witt ring also has a natural $q$-deformation, which was studied in [20], [21], [22]. These and other $q$-deformations of Witt rings will be crucial to our analysis of possible $q$-deformations of the Bost-Connes system. We discuss these $q$-deformations in the next section, and other forms of "geometric" $q$-deformations in the following sections.

\section{Witt Rings and their $q$-Deformations}

2.1. Witt rings and operations. We first recall some basic definitions regarding Witt rings. For $A$ an associative commutative ring, let End $_{A}$ be the category of endomorphisms of projective $A$-modules of finite rank. The objects of this category are pairs $(E, f)$, where $f \in \operatorname{End}_{A}(E)$. With the direct sum and the tensor product defined componentwise on the objects, the Grothendieck group $K_{0}\left(\operatorname{End}_{A}\right)$ also acquires a commutative ring structure. Let $K_{0}(A)$ be the ideal generated by the pairs of the form $(E, f=0)$. Then one defines

$$
W_{0}(A)=K_{0}\left(\underline{\operatorname{End}_{A}}\right) / K_{0}(A) .
$$

There are several operators defined on $W_{0}(A)$, including the Frobenius, the Verschiebung, and the Teichmüller and ghost maps, [1]. These play an important role in the relation between Witt rings and the integral Bost-Connes system, as shown in [5].

The ghost ring functor $g h:$ Rings $\rightarrow$ Rings associates to each object $A$ the ring whose underlying set is

$$
A^{\infty}:=\left\{\left(x_{n}\right)_{n \in \mathbb{N}} \mid x_{n} \in A\right\}
$$

and to each morphism $f: A \rightarrow B$ the morphism

$$
\operatorname{gh}(f):\left(x_{n}\right)_{n \in \mathbb{N}} \mapsto\left(f\left(x_{n}\right)\right)_{n \in \mathbb{N}} .
$$

Given an associative and commutative ring A, the Big Witt Ring $W(A)$ is characterized by the three properties:

(1) As a set, $W(A)$ equals $A^{\infty}$.

(2) For any ring homomorphism $f: A \rightarrow B$, the map $W(f): W(A) \rightarrow W(B)$ given by

$$
\left(x_{n}\right)_{n \in \mathbb{N}} \mapsto\left(f\left(x_{n}\right)\right)_{n \in \mathbb{N}}
$$

is a ring homomorphism. 
(3) The map $\Phi: W(A) \rightarrow \operatorname{gh}(A)$, given by

$$
\left(x_{n}\right)_{n \in \mathbb{N}} \mapsto\left(\sum_{d \mid n} d x_{d}^{\frac{n}{d}}\right)_{n \in \mathbb{N}},
$$

is a ring homomorphism, where $\operatorname{gh}(A)$ is the image of $A$ under the ghost ring functor described above.

The Frobenius, Verschiebung, Teichmüller, and ghost map operators are also defined on the ring $W(A)$, satisfying the same set of relations as on $W_{0}(A)$, see [1].

We also recall briefly the relation between $W_{0}(A)$ and $W(A)$, and the Grothendieck $\lambda$-ring $\Lambda(A)$, see [1, 24].

Given an element $(E, f)$ in $W_{0}(A)$, let $M(f)$ denote the matrix representation of $f: E \rightarrow$ $E$, as in [1], [5]. The following properties determine a natural embedding of $W_{0}(A)$ into $W(A)$, see [1].

- The map $L: W_{0}(A) \rightarrow \Lambda(A)$ given by $L(E, f) \mapsto \operatorname{det}(1-t M(f))^{-1}$ is a homomorphic injection into the additive group of the $\Lambda$ ring, with image given by the subgroup

$$
\operatorname{Range}(L)=\left\{\frac{1+a_{1} t+\ldots+a_{n} t^{n}}{1+b_{1} t+\ldots+b_{m} t^{m}}, a_{i}, b_{j} \in A\right\} .
$$

- The Artin-Hasse exponential map $\varepsilon: W(A) \rightarrow \Lambda(A)$ given by

$$
\varepsilon:\left(x_{n}\right) \mapsto \prod_{n \in \mathbb{N}} \frac{1}{1-x_{n} t^{n}}
$$

is an isomorphism.

2.2. q-Deformations of Witt rings. $q$-Deformations of the Witt Ring, considered as a $q$ deformed functor from the category of rings to itself, and of the ghost map were introduced in [20], 21], 22]. We recall the basic properties of these deformations.

For $q$ a positive integer and $A$ an associative commutative ring, the $q$-deformed Big Witt Ring $W^{q}(A)$ is characterized in [22] by the following three conditions:

(1) As a set, $W^{q}(A)$ equals $A^{\infty}$

(2) For any ring homomorphism $f: A \rightarrow B$, the map $W^{q}(f): W^{q}(A) \rightarrow W^{q}(B)$

$$
\left(x_{n}\right)_{n \in \mathbb{N}} \mapsto\left(f\left(x_{n}\right)\right)_{n \in \mathbb{N}}
$$

is a ring homomorphism.

(3) The map $\Phi^{q}: W(A) \rightarrow g h(A)$

$$
\left(x_{n}\right)_{n \in \mathbb{N}} \mapsto\left(\sum_{d \mid n} d q^{\frac{n}{d}-1} x_{d}^{\frac{n}{d}}\right)_{n \in \mathbb{N}}
$$

is a ring homomorphism.

Moreover, the Grothendieck $\lambda$-ring also admits a $q$-deformation $\Lambda^{q}(A)$, as in [21. As a set, $\Lambda^{q}(A)=1+t A[t]=\left\{1+\sum_{n=1}^{\infty} a_{n} t^{n}, a_{n} \in A, n \geq 1\right\}$, where the addition is defined as the usual multiplication of power series raised to the $q$-th power, and the multiplication is defined by requiring that

$$
(1-a t)^{-q} \star_{q}(1-b t)^{-q}=(1-a b t)^{-q}
$$


It is proved in [20] that, for every commutative ring in which $q$ is invertible, the map $\eta: \Lambda(A) \rightarrow \Lambda^{q}(A)$, given by $\eta(f)=f(t)^{q}$, is an isomorphism. Thus, in this case, one can identify the underlying set of $\Lambda^{q}(A)$ with the $q$-th powers of the power series in $t$ with coefficients in $A$ having constant term equal to 1.

Note that the product (2.4) under $\star_{q}$ in $\Lambda^{q}(A)$ differs from the usual product $(1-a t)^{-q} \star$ $(1-b t)^{-q}=(1-a b t)^{-q^{2}}$ as elements of $\Lambda(A)$. In particular, for $A=k$, while the product of $(1-a t)^{-q}$ and $(1-b t)^{-q}$ in $\Lambda(k)$ can be interpreted as $L\left(\left(E_{1}^{\oplus q}, f_{1}^{\oplus q}\right) \otimes\left(E_{2}^{\oplus q}, f_{2}^{\oplus q}\right)\right)=$ $(1-a b t)^{-q^{2}}$ for $\left(E_{1}, f_{1}\right)=(k, a)$ and $\left(E_{2}, f_{2}\right)=(k, b)$, the corresponding identity

$$
L^{q}(k, a) \star_{q} L^{q}(k, b)=L^{q}(k, a b)
$$

that matches (2.4) does not correspond to just identifying $L^{q}(E, f)$ with $L\left(E^{\oplus q}, f^{\oplus q}\right)$.

Proposition 2.1. Let $A$ be a commutative ring in which $q$ is invertible. The q-deformed characteristic polynomial

$$
L^{q}: W_{0}(A) \rightarrow \Lambda^{q}(A), \quad L^{q}(E, f)=\operatorname{det}(1-t M(f))^{-q}
$$

determines a homomorphic injection whose image is

$$
\operatorname{Range}\left(L^{q}\right)=\left\{\frac{\left(1+a_{1} t+\ldots+a_{n} t^{n}\right)^{q}}{\left(1+b_{1} t+\ldots+b_{m} t^{m}\right)^{q}}, a_{i}, b_{j} \in A\right\} .
$$

Proof. The result of [20] mentioned above, showing that the map $\eta(f)=f(t)^{q}$ is an isomorphism, combined with the homomorphic injection $L(E, f) \mapsto \operatorname{det}(1-t M(f))^{-1}$ to (2.2), implies that the following diagram commutes

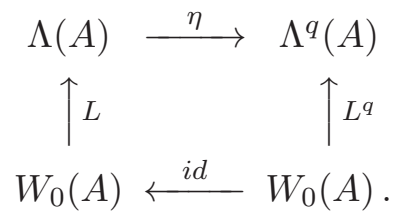

The result then follows.

Corollary 2.2. The q-deformed Witt ring $W^{q}(A)$ contains an isomorphic copy of $W_{0}(A)$.

Proof. The $q$-deformed Witt ring $W^{q}(A)$ is isomorphic to $\Lambda^{q}(A)$ through a $q$-analog of the Artin-Hasse map, see [23]. It is then easy to see that $W_{0}(A)$ can be embedded inside $W^{q}(A)$, with the map given by the composition of the $q$-deformed characteristic polynomial (2.5) with the $q$-analog of the Artin-Hasse map.

Later, we will describe an explicit canonical embedding of $W_{0}(A)$ into $W^{q}(A)$, in the special case where $A=k$ is an algebraically closed field.

\section{Undeformed Bost-Connes Algebras in $q$-DEFormed WitT RINGS}

The integral form of the Bost-Connes algebra introduced in [7] is directly related to the Witt ring $W_{0}(A)$, as shown in [5]. The operations $\sigma_{n}$ and $\tilde{\rho}_{n}$ of the integral Bost-Connes algebra are induced by the Frobenius and Verschiebung and their extensions to the Witt ring $W(A)$. In this section, we consider the $q$-embedding of $W_{0}(A)$ into $W^{q}(A)$ of Corollary 2.2. and the natural operations in $W^{q}(A)$, and we show that the same construction of [5] goes over with minor modifications to this case and realize the same (undeformed) integral Bost-Connes algebra in terms of Frobenius and Verschiebung on the $q$-deformed Witt ring 
$W^{q}(A)$. We will see in the following section how this suggests then a natural deformation of the integral Bost-Connes algebra, based on a modification of the $q$-deformation $W^{q}(A)$ of the Witt ring, which differs from the one introduced in [20, [21], [22], 223].

3.1. Operations on q-deformed Witt rings. We begin by checking that operations and relations on $W_{0}(A)$ extend compatibly to $W^{q}(A)$ through the $q$-embedding.

As mentioned in 2.1 , the ghost map, the Frobenius, and the Verschiebung are operators defined on $W_{0}(A)$, and $W(A)$. It is shown in [1] that the operators defined on them are, in the appropriate sense, compatible. We now prove that the operators defined on $W_{0}(A)$, when the latter is $q$-embedded in $W^{q}(A)$, are also compatible with the operators defined on $W^{q}(A)$.

Definition 3.1. Let $A^{q}[t]$ be the ring whose underlying set is $A[t]$, with the addition defined by the usual addition of power series, and the multiplication defined by

$$
\sum a_{n} t^{n} \sum b_{n} t^{n}=\sum \frac{1}{q}\left(a_{n} b_{n}\right) t^{n} .
$$

In the following we focus on the case where $A=k$ is an algebraically closed field. We obtain the following compatibilities between $W_{0}(k)$ and $W^{q}(k)$ under the $q$-embedding.

Proposition 3.2. Let $A=k$ be an algebraically closed field. The following compatibilities hold between operations on $W_{0}(k)$ and $W^{q}(k)$ under the q-embedding of Corollary 2.2.

(1) the ghost map $g h_{n}$ defined on $W_{0}(k)$ by

$$
g h_{n}: W_{0}(k) \rightarrow g h^{\prime}(k), \quad(E, f) \mapsto\left(\operatorname{tr}\left(f^{n}\right)\right)_{n \in \mathbb{N}}
$$

is compatible with the ghost map defined on $W^{q}(k)$ by $\Phi^{q}$ of (2.3), in the sense that the following diagram commutes:

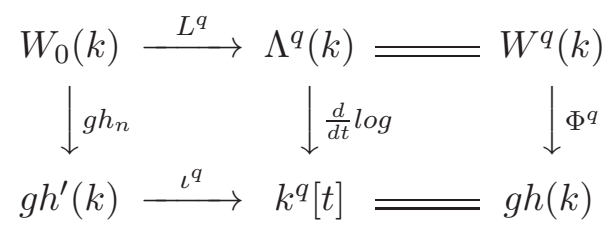

where the q-identification $\iota^{q}$ is given by

$$
\iota^{q}:\left(x_{n}\right) \mapsto \sum_{n} q x_{n} t^{n-1} .
$$

(2) The Frobenius map $F_{n}$ defined on $W_{0}(k)$ by $(E, f) \rightarrow\left(E, f^{n}\right)$ is compatible with the Frobenius map $F_{n}^{\prime}$ on $W^{q}(k)$ in the sense that the following diagram is commutative:

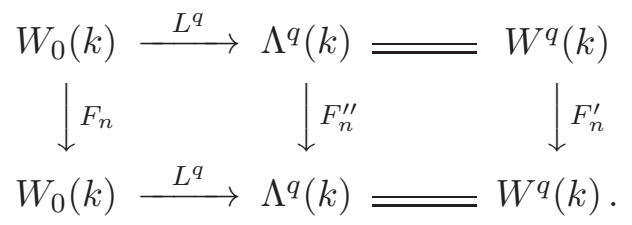

(3) The Verschiebung operator $V_{n}$ defined on $W_{0}(k)$ by

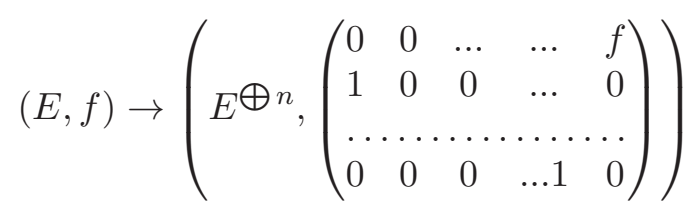


is compatible with the Verschiebung operator $V_{n}^{\prime}$ on $W^{q}(k)$ in the sense that the following diagram is commutative:

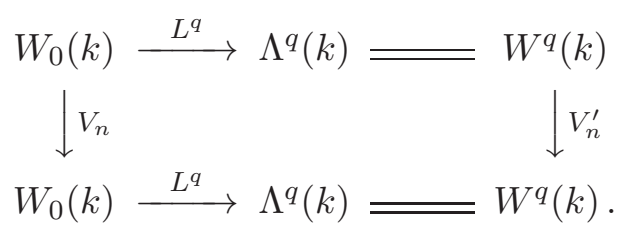

Proof. We work under the assumption that $A=k$ is an algebraically closed field.

(1) It is clear from [1] and 23] that the right half of the diagram is commutative. The isomorphism between $\Lambda^{q}(k)$ and $W^{q}(k)$ is given by the $q$-deformed ArtinHasse exponential map. The isomorphism between $g h(k)$ and $k^{q}[t]$ is given by the $q$-identification (3.2). In an algebraically closed field, the matrix associated with the endomorphism $f$ can be triangulated. Therefore, the $n$-th component of the ghost map is given by $\sum_{i} \alpha_{i}^{n}$, where $\alpha_{i}$ are the eigenvalues associated with the endomorphism $f$. Note that, given these eigenvalues,

$$
L^{q}(E, f)=\operatorname{det}(1-t f)^{-q}=\prod_{i}\left(1-\alpha_{i}\right)^{-q} .
$$

Taking the log-derivative of this, we then obtain

$$
\frac{d}{d t} \log \left(\prod_{i}\left(1-\alpha_{i}\right)^{-q}\right)=q \frac{d}{d t} \sum_{i} \log \left(1-\alpha_{i}\right)^{-1}=q \sum_{j}^{\infty}\left(\sum_{i} \alpha_{i}^{j}\right) t^{j-1},
$$

where the second identify follows from the identity

$$
\frac{d}{d t} \log \left(\frac{1}{1-a t}\right)=\sum_{i} a^{i} t^{i-1}
$$

which is obtained from the Taylor expansion of the log function. Given the $i$-th ghost component of $W_{0}(k)$, which is $x_{i}=\sum_{i} \alpha_{i}^{j}$, we obtain through the $q$-identification,

$$
\iota^{q}\left(\left(x_{n}\right)\right)=q \sum_{j}^{\infty}\left(\sum_{i} \alpha_{i}^{j}\right) t^{j-1} .
$$

(2) As in [1, using the identification between $W^{q}(k)$ and the ring $\Lambda^{q}(k)$, we see that the Frobenius map on $W^{q}(k)$ is the same as the map

$$
F_{n}^{\prime \prime}:(1-a t)^{-1} \mapsto\left(1-a^{n} t\right)^{-1}
$$

Then, suppose given $(E, f) \in W_{0}(k)$, where $f$ has eigenvalues $\alpha_{i}$. The Frobenius map sends $(E, f)$ to $\left(E, f^{n}\right)$, where the eigenvalues of $f^{n}$ ware $\alpha_{i}^{n}$. Then we obtain

$$
F_{n}^{\prime \prime}\left(L^{q}(E, f)\right)=F_{n}^{\prime \prime}\left(\prod_{i}\left(1-\alpha_{i}\right)^{-q}\right)=\prod_{i}\left(1-\alpha_{i}^{n}\right)^{-q}=L^{q}\left(E, f^{n}\right)=L^{q}\left(F_{n}(E, f)\right) .
$$

(3) Again, the equivalence of the right half of the diagram is shown in [23. Note that the Verschiebung operator $V_{n}$ acting on $\Lambda^{q}(k)$ is defined by

$$
V_{n}:(1-a t)^{-1} \mapsto\left(1-a t^{n}\right)^{-1},
$$


for $(1-a t)^{-1} \in \Lambda^{q}(k)$. A direct calculation then shows that

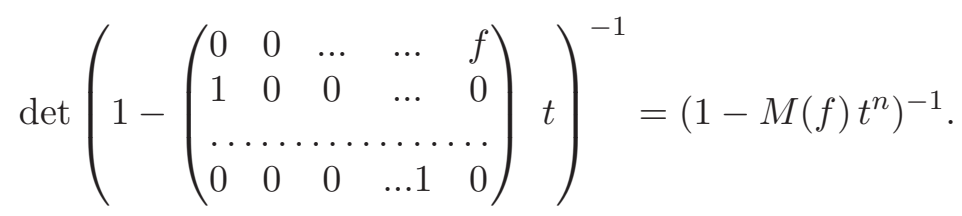

Thus, the commutativity of the left half of the diagram also follows.

3.2. Divisor map and undeformed Bost-Connes algebra. When $A$ is an algebraically closed field $k$, the determinant function factors completely into linear forms in terms of the eigenvalues of the endomorphisms. Associating to each pair $(E, f)$ the divisor $\delta(f)$ of nonzero eigenvalues of $f$ determines a ring isomorphism between $W_{0}(k)$ and the group ring $\mathbb{Z}\left[k^{*}\right]$. If in particular $k=\overline{\mathbb{F}}_{p}$ is the algebraic closure of a finite field $\mathbb{F}_{p}$, it is shown in [5] that one obtains a natural isomorphism

$$
\sigma: W_{0}\left(\overline{\mathbb{F}}_{p}\right) \rightarrow \mathbb{Z}\left[(\mathbb{Q} / \mathbb{Z})^{(p)}\right],
$$

where $(\mathbb{Q} / \mathbb{Z})^{(p)}$ is the group of fractions with denominator prime to $p$.

The map $\sigma$ of (3.4) is induced by the divisor map. Given an element $(E, f)$ in $W_{0}(k)$ with $\alpha$ the eigenvalues of $f$ and $n(\alpha)$ their multiplicities, the divisor map is given by

$$
\delta(f):=\delta(L(E, f))=\delta\left(\prod(1-\alpha t)^{-n(\alpha)}\right)=\sum n(\alpha)[\alpha],
$$

As shown in Proposition 2.3 of [5], it defines an element in $\mathbb{Z}\left[k^{*}\right]$, and one obtains a ring isomorphism

$$
\delta: W_{0}(k) \rightarrow \mathbb{Z}\left[k^{*}\right] .
$$

When we consider $W_{0}(k)$ as embedded in the $q$-deformed Witt ring $W^{q}(k)$, or equivalently we use the $q$-deformed characteristic polynomial $L^{q}: W_{0}(k) \rightarrow \Lambda^{q}(k)$, the divisor map (3.5) is no longer compatible with the multiplication $\star_{q}$ in $\Lambda^{q}(k)$. Indeed, we have

$$
\delta\left(L^{q}(E, f)\right)=\delta\left(\prod(1-\alpha t)^{-q n(\alpha)}\right)=\sum q n(\alpha)[\alpha] .
$$

Using $(k, a) \otimes(k, b)=(k, a b)$, we have $L^{q}(k, a)=(1-a t)^{-q}$ and $L^{q}(k, b)=(1-b t)^{-q}$ in $\Lambda^{q}(k)$ with $L^{q}((k, a) \otimes(k, b))=L^{q}(k, a) \star_{q} L^{q}(k, b)=(1-a b t)^{-q}=L^{q}(k, a b)$, by the definition of the induced product $\star_{q}$ on $\Lambda^{q}(k)$, while the multiplication of the divisors as elements in the group ring gives $\delta\left(L^{q}(k, a)\right) \delta\left(L^{q}(k, b)\right)=\left(\sum q n(\alpha)[\alpha]\right)\left(\sum q n(\beta)[\beta]\right)=q \cdot \delta\left(L^{q}(k, a b)\right)$.

Thus, the only way to restore the multiplicative property is to compute $\delta_{q}\left(L^{q}(E, f)\right)=$ $q^{-1} \delta\left(L^{q}(E, f)\right)=\delta(L(E, f))$. This restores the original map (3.5) on the undeformed $\Lambda(k)$, hence the same undeformed Bost-Connes algebra as constructed in [5].

While the $q$-deformations of Witt rings considered in [20, 21], [22], 223] do not directly lead to a $q$-deformed Bost-Connes algebra through the same construction of [5], this suggests a modification of the construction of $q$-deformed Witt rings, with a different motivation in mind than the $q$-Möbius functions and $q$-deformed necklace rings that motivated the construction of [20], 21], 222, 23]. 


\section{Geometric $q$-Deformations of Witt Rings}

In this section we discuss a different approach to $q$-deforming the Witt rings, and we show that, unlike the case discussed in the previous section, this leads to $q$-deformations of the Bost-Connes algebra. The crucial difference here is that, instead of $q$-deforming the Witt ring $W(A)$ to $W^{q}(A)$, or equivalently deforming $\Lambda(A)$ to $\Lambda^{q}(A)$ as in [20, 21, [22, [23, we consider a deformation of $W_{0}(A)$ to a $q$-deformed $\mathcal{W}_{0}^{q}(A)$, while we maintain the product in $W(A)$ and $\Lambda(A)$ undeformed.

Geometrically, if we consider elements of $\Lambda(k)$ that arise from zeta functions of schemes (see [24]), the two kinds of deformations that we introduce in this section have a very simple geometric meaning. The first arises by replacing a scheme $X$ with a tower where $X_{q^{\ell}}=X \sqcup \cdots \sqcup X$, a disjoint union of $q^{\ell}$ copies of $X$ (or equivalently the product of $X$ with a $q^{\ell}$ points). The second deformation consists of replacing $X$ with the tower of the products $X \times \mathbb{A}^{\ell}$. Since, when $q$ is a prime power $q=p^{r}$, the number of points $\# A^{\ell}\left(\mathbb{F}_{q}\right)=q^{\ell}$, the second construction will be an extension of the first, where the case of $q^{\ell}$-points appears as the first term, but all the additional contributions of the field extensions $\mathbb{A}^{\ell}\left(\mathbb{F}_{q^{n}}\right)$ are also counted. Thus, we refer to them, respectively, as the $q^{\ell}$-points deformation and the $\mathbb{A}^{\ell}$-deformation.

4.1. The $q^{\ell}$-points deformation. Let $\Omega_{q}$ denote the map $\Omega_{q}: W_{0}(A) \rightarrow W_{0}(A)$ that maps $\Omega_{q}:(E, f) \mapsto\left(E^{\oplus q}, f^{\oplus q}\right)$. Consider the restriction of the characteristic polynomial map $L: W_{0}(A) \rightarrow \Lambda(A)$ to the range $\Omega_{q}\left(W_{0}(A)\right)$. Notice that, unlike the $q$-deformations considered in the previous sections, here we do not deform the product in $\Lambda(A)$. We work with the undeformed product determined by

$$
(1-a t)^{-1} \star(1-b t)^{-1}=(1-a b t)^{-1} .
$$

We define $S^{q}(E, f)=L\left(\Omega_{q}(E, f)\right)$. Note that this is the same characteristic polynomial (1$t M(f))^{-q}$, as in the case of the $q$-deformed characteristic polynomial $L^{q}(E, f)$ considered above, except that now we regard it as an element of the undeformed ring $\Lambda(A)$ rather than as an element of $\Lambda^{q}(A)$.

Definition 4.1. The $q^{\ell}$-points deformation of the Witt ring $W_{0}(A)$ is the graded ring $\mathcal{W}_{0}^{q}(A)$ defined as a set by

$$
\mathcal{W}_{0}^{q}(A)=\oplus_{\ell \geq 0} \Omega_{q^{\ell}}\left(W_{0}(A)\right),
$$

with addition and multiplication induced uniquely by addition and multiplication in $W_{0}(A)$.

Lemma 4.2. The multiplication operation on $\mathcal{W}_{0}^{q}(A)$ obtained as above satisfies

$$
\left.\star: \Omega_{q^{\ell}}\left(W_{0}(A)\right) \times \Omega_{q^{\ell^{\prime}}}\left(W_{0}(A)\right) \rightarrow \Omega_{q^{\ell+\ell^{\prime}}}\left(W_{0}(A)\right)\right) .
$$

Proof. We have $\Omega_{q^{\ell}}\left(E_{1}, f_{1}\right)=\left(E_{1}^{\otimes q^{\ell}}, f_{1}^{\otimes q^{\ell}}\right)$ and $\Omega_{q^{\ell^{\prime}}}\left(E_{2}, f_{2}\right)=\left(E_{2}^{\otimes q^{\ell^{\prime}}}, f_{2}^{\otimes q^{\ell^{\ell^{\prime}}}}\right)$, hence their product in $W_{0}(A)$ is given by $\left(\left(E_{1} \otimes E_{2}\right)^{\oplus q^{\ell+\ell^{\prime}}},\left(f_{1} \otimes f_{2}\right)^{\oplus q^{\ell+\ell^{\prime}}}\right)$ and we obtain

$$
\Omega_{q^{\ell}}\left(E_{1}, f_{1}\right) \otimes \Omega_{q^{\ell^{\prime}}}\left(E_{2}, f_{2}\right)=\Omega_{q^{\ell+\ell^{\prime}}}\left(E_{1} \otimes E_{2}, f_{1} \otimes f_{2}\right) .
$$


At the level of characteristic polynomials this corresponds to the product relation

$$
\left.S^{q^{\ell}}\left(E_{1}, f_{1}\right) \star S^{q^{\ell^{\prime}}}\left(E_{2}, f_{2}\right)=S^{q^{\ell+\ell^{\prime}}}\left(E_{1} \otimes E_{2}, f_{1} \otimes f_{2}\right)\right)
$$

since we have

$$
\begin{aligned}
L\left(\Omega_{q^{\ell}}\left(E_{1}, f_{1}\right)\right) \star & \left.L\left(\Omega_{q^{\ell^{\prime}}}\left(E_{2}, f_{2}\right)\right)\right)=L\left(\Omega_{q^{\ell}}\left(E_{1}, f_{1}\right) \otimes \Omega_{q^{\ell^{\prime}}}\left(E_{2}, f_{2}\right)\right) \\
& =L\left(\Omega_{q^{\ell+\ell^{\prime}}}\left(E_{1} \otimes E_{2}, f_{1} \otimes f_{2}\right)\right) .
\end{aligned}
$$

Lemma 4.3. The Frobenius $F_{n}$ and Verschiebung $V_{n}$ on $W_{0}(A)$ extend to a Frobenius $F_{n}$ and Verschiebung $V_{n}$ on $\mathcal{W}_{0}^{q}(A)$, satisfying $\Omega_{q^{\ell}} \circ F_{n}=F_{n} \circ \Omega_{q^{\ell}}$ and $\Omega_{q^{\ell}} \circ V_{n}=\tilde{V}_{n} \circ \Omega_{q^{\ell}}$, where $\tilde{V}_{n}$ is $V_{n}$ up to a a change of basis given by a permutation.

Proof. We have $F_{n}(E, f)=\left(E, f^{n}\right)$, hence $F_{n}\left(E^{\oplus q^{\ell}}, f^{\oplus q^{\ell}}\right)=\left(E^{\oplus q^{\ell}},\left(f^{n}\right)^{\oplus q^{\ell}}\right)=\Omega_{q^{\ell}}\left(E, f^{n}\right)$. We index the entries of the matrix

$$
V_{n}(f)=\left(\begin{array}{ccccc}
0 & 0 & \ldots & \ldots & f \\
1 & 0 & 0 & \ldots & 0 \\
\ldots & \ldots \ldots \ldots \ldots & \ldots \\
0 & 0 & 0 & \ldots 1 & 0
\end{array}\right)
$$

as $\left(V_{n}(f)\right)_{i j}$ with $i=(a, b)$ and $j=\left(a^{\prime}, b^{\prime}\right)$ where $a, a^{\prime}=1, \ldots, k$ where $k \times k$ is the dimension of $M(f)$, and $b, b^{\prime}=1, \ldots, n$. The second indices $\left(b, b^{\prime}\right)$ specify which square block of size $k$ we are considering and the first index $\left(a, a^{\prime}\right)$ locates the position in that square block. Then the entries of $V_{n}(f)$ are

$$
\left(V_{n}(f)\right)_{i j}= \begin{cases}f_{a a^{\prime}} & b=1, b^{\prime}=q \\ 1 & a=a^{\prime}, b^{\prime}=b-1 \\ 0 & \text { otherwise. }\end{cases}
$$

Thus, $V_{n}\left(f^{\oplus q^{\ell}}\right)$ can be indexed by $\left(V_{n}\left(f^{\oplus q^{\ell}}\right)\right)_{i j}$ with $i=(a, b, r)$ and $j=\left(a^{\prime} b^{\prime}, r^{\prime}\right)$ with $a, a^{\prime}, b, b^{\prime}$ as above and $r, r^{\prime}=1, \ldots q^{\ell}$ with entries as above for $r=r^{\prime}$ and zero otherwise. Clearly, this also indexes the entries of $\left(V_{n}(f)\right)^{\oplus q^{\ell}}$.

We use the same notation $F_{n}$ and $V_{n}$ for the Frobenius and Verschiebung on $\mathcal{W}_{0}^{q}(A)$.

Proposition 4.4. Let $k$ be an algebraically closed field. The divisor map (3.5) extends to a ring isomorphism $\delta_{q}: \mathcal{W}_{0}^{q}(k) \rightarrow \mathbb{Z}[q]\left[k^{*}\right]$ given by

$$
\delta_{q}\left(\Omega_{q^{\ell}}(E, f)\right)=q^{\ell} \delta(L(E, f))=q^{\ell} \sum n(\alpha)[\alpha] .
$$

Proof. We have

$$
\begin{gathered}
\delta_{q}\left(\Omega_{q^{\ell}}(A, f)\right)=\delta\left(S^{q^{\ell}}(A, f)\right)=\delta\left(\prod(1-\alpha t)^{-q^{\ell} n(\alpha)}\right) \\
=q^{\ell} \sum n(\alpha)[\alpha] .
\end{gathered}
$$

It is clear that $\delta_{q}$ is compatible with addition, and by (4.3) it is also compatible with multiplication

$$
\begin{gathered}
\delta_{q}\left(\Omega_{q^{\ell+\ell^{\prime}}}\left(E_{1} \otimes E_{2}, f_{1} \otimes f_{2}\right)\right)=\delta_{q}\left(\Omega_{q^{\ell}}\left(E_{1}, f_{1}\right) \otimes \Omega_{q^{\ell^{\prime}}}\left(E_{2}, f_{2}\right)\right) \\
\left.=\delta\left(S^{q^{\ell}}\left(E_{1}, f_{1}\right) \star S^{q^{\ell^{\prime}}}\left(E_{2}, f_{2}\right)\right)=\delta\left(S^{q^{\ell+\ell^{\prime}}}\left(E_{1} \otimes E_{2}, f_{1} \otimes f_{2}\right)\right)\right) \\
=q^{\ell+\ell^{\prime}} \sum n(\alpha) n(\beta)[\alpha][\beta]=\left(q^{\ell} \sum n(\alpha)[\alpha]\right)\left(q^{\ell^{\prime}} \sum n(\beta)[\beta]\right) \in \mathbb{Z}[q]\left[k^{*}\right] .
\end{gathered}
$$

The fact that the original $\delta$ is a bijection also implies that $\delta_{q}$ is a bijection. 
Using as in [5] an isomorphism $\sigma: \overline{\mathbb{F}}_{p}^{*} \rightarrow(\mathbb{Q} / \mathbb{Z})^{(p)}$ together with the divisor map $\delta_{q}$ of (4.5) we obtain an isomorphism

$$
\tilde{\sigma}_{q}: \mathcal{W}_{0}^{q}\left(\overline{\mathbb{F}}_{p}\right) \rightarrow \mathbb{Z}[q]\left[(\mathbb{Q} / \mathbb{Z})^{(p)}\right], \quad \tilde{\sigma}_{q}=\sigma \circ \delta_{q} .
$$

Note that the construction of the deformation $\mathcal{W}_{0}^{q}\left(\overline{\mathbb{F}}_{p}\right)$ and the divisor map (4.6) make sense for an arbitrary integer $q$, which is not necessarily a power of $p$.

4.2. The $q^{\ell}$-points deformation of the Bost-Connes algebra. Let $\tilde{\sigma}_{q}: \mathcal{W}_{0}^{q}\left(\bar{F}_{p}\right) \rightarrow$ $\mathbb{Z}[q]\left[(\mathbb{Q} / \mathbb{Z})^{(p)}\right] \subset \mathbb{Z}[q][\mathbb{Q} / \mathbb{Z}]$ be as in (4.6). We consider endomorphisms $\sigma_{n, q}$ of $\mathbb{Z}[q][\mathbb{Q} / \mathbb{Z}]$ that satisfy

$$
\sigma_{n, q} \circ \tilde{\sigma}_{q}=\tilde{\sigma}_{q} \circ F_{n},
$$

with $F_{n}$ the Frobenius on $\mathcal{W}_{0}^{q}\left(\overline{\mathbb{F}}_{p}\right)$.

Lemma 4.5. Let $E(r, k):=q^{k} e(r)$ in $\mathbb{Z}[q][\mathbb{Q} / \mathbb{Z}]$, where $e(r)$ are the generators of $\mathbb{Z}[\mathbb{Q} / \mathbb{Z}]$. The endomorphisms $\sigma_{n, q}: \mathbb{Z}[q][\mathbb{Q} / \mathbb{Z}] \rightarrow \mathbb{Z}[q][\mathbb{Q} / \mathbb{Z}]$ satisfying (4.7) are given by

$$
\sigma_{n, q}(E(r, k))=q^{k} e(n r)=E(n r, k) .
$$

Proof. Given $\Omega_{q^{k}}(E, f) \in \mathcal{W}_{0}^{q}(A)$, with $L(E, f)=\prod(1-\alpha t)^{-n(\alpha)}$ we have $\delta_{q}\left(\Omega_{q^{k}}(E, f)\right)=$ $q^{k} \sum n(\alpha)[\alpha]$, while $\delta_{q}\left(F_{n}\left(\Omega_{q^{k}}(E, f)\right)=q^{k} \sum n(\alpha)\left[\alpha^{n}\right]\right.$.

Thus, we see that, with this choice of $q^{\ell}$-points deformation $\mathcal{W}_{0}^{q}\left(\bar{F}_{p}\right)$ the resulting $q$ deformation of the Bost-Connes algebra is essentially trivial, consisting only of replacing the coefficient ring $\mathbb{Z}$ of $\mathbb{Z}[\mathbb{Q} / \mathbb{Z}]$ by the polynomial ring $\mathbb{Z}[q]$, but the operations $\sigma_{n, q}$ (hence also the corresponding operations $\tilde{\rho}_{n, q}$ ) remain the same unperturbed operation of the original Bost-Connes algebra and are the identity on the polynomial ring $\mathbb{Z}[q]$.

Corollary 4.6. The deformation of the Bost-Connes algebra $\mathcal{A}_{\mathbb{Z}}$ induced by the $q^{\ell}$-points deformation $\mathcal{W}_{0}^{q}\left(\bar{F}_{p}\right)$ of the Witt ring $W_{0}\left(\overline{\mathbb{F}}_{p}\right)$ is simply given by the product $\mathcal{A}_{\mathbb{Z}} \otimes_{\mathbb{Z}} \mathbb{Z}[q]$.

4.3. The $\mathbb{A}^{\ell}$-deformation. We improve on the construction described above by replacing the $q^{\ell}$-points deformation with another geometrically motivated deformation, which arises from thinking of the $q^{\ell}$ points as the $\mathbb{F}_{q}$ points of an affine space $\mathbb{A}^{\ell}$ and constructing the deformation determined geometrically by taking products with affine spaces.

Recall that, if $X$ is a scheme of finite type over $k=\mathbb{F}_{q}$ with $q=p^{r}$ a prime power, then the associated zeta function is given by

$$
Z(X, t)=\exp \left(\sum_{m \geq 1} N_{m}(X) \frac{t^{m}}{m}\right)=\prod_{x}\left(1-t^{\operatorname{deg}(x)}\right)^{-1},
$$

with $N_{m}(X)=\# X\left(F_{q^{m}}\right)$ and $\operatorname{deg}(x)$ the degree of the extension $\left[k(x): \mathbb{F}_{q}\right]$, with $k(x)$ the residue field at the point $x$. Writing $N_{m}(X)=\sum_{r \mid m} r \cdot a_{r}$, where

$$
a_{r}=\#\left\{x:\left[k(x): \mathbb{F}_{q}\right]=r\right\} \text {, }
$$

we obtain

$$
Z(X, t)=\prod_{r \geq 1}\left(1-t^{r}\right)^{-a_{r}}
$$


In the case of an affine space $\mathbb{A}^{\ell}$ we have

$$
Z\left(\mathbb{A}^{\ell}, t\right)=\exp \left(\sum_{m} q^{\ell m} \frac{t^{m}}{m}\right)=\left(1-q^{n} t\right)^{-1}
$$

In terms of (4.9) this is

$$
Z\left(\mathbb{A}^{\ell}, t\right)=\left(1-q^{n} t\right)^{-1}=\prod_{r \geq 1}\left(1-t^{n}\right)^{-M\left(q^{n}, r\right)},
$$

where

$$
M\left(q^{n}, r\right)=\frac{1}{r} \sum_{d \mid r} \mu(d) q^{\frac{n r}{d}},
$$

with $\mu(x)$ the Möbius function.

Given a scheme $X$ with zeta function $Z(X, t)$, taking the product $X \times \mathbb{A}^{\ell}$ gives

$$
Z\left(X \times \mathbb{A}^{\ell}, t\right)=Z\left(X, q^{\ell} t\right) .
$$

More generally, as discussed in [24], one should regard zeta functions $Z(X, t)$ as elements in the Witt ring $W(\mathbb{Z})$ satisfying

$$
Z(X \times Y, t)=Z(X . t) \star Z(Y, t),
$$

where $\star$ is the Witt ring product determined by $(1-a t)^{-1} \star(1-b t)^{-1}=(1-a b t)^{-1}$. For a disjoint union $X \sqcup Y$, the zeta functions multiply, where multiplication of series corresponds to the addition operation $+{ }_{w}$ in the Witt ring,

$$
Z(X \sqcup Y, t)=Z(X, t) \cdot Z(Y, t)=Z(X, t)+{ }_{w} Z(Y, t) .
$$

Indeed, a more general inclusion-exclusion formula holds, see [24].

Remark 4.7. Since $M(q, 1)=q$, we have

$$
(1-q t)^{-1}=(1-t)^{-q} \cdot \prod_{r>1}\left(1-t^{r}\right)^{-M(q, r)} .
$$

The first term $(1-t)^{-q}$ is the kind of $q$-deformation of $(1-t)^{-1}$ that we considered in the previous sections. In this sense, we can regard $(1-q t)^{-1}$ as a generalization of the $q$-deformation $(1-t)^{-q}$ discussed before, where the previous deformation appears as the order one term.

Modeled on the behavior of these geometric zeta functions, we consider a different construction of a $q$-deformation of the Witt ring $W_{0}(A)$. For $(E, f) \in W_{0}(A)$ with $L(E, f)=$ $\prod(1-\alpha t)^{-n(\alpha)}$, let

$$
\tilde{\Omega}_{q^{\ell}}(L(E, f)):=\prod\left(1-\alpha q^{\ell} t\right)^{-n(\alpha)} .
$$

Definition 4.8. The $\mathbb{A}^{\ell}$-perturbation of the Witt ring $W_{0}(A)$ is the graded ring $\tilde{\mathcal{W}}_{0}^{q}(A)$ defined as a set by

$$
\tilde{\mathcal{W}}_{0}^{q}(A)=\oplus_{\ell \geq 0} \tilde{\Omega}_{q^{\ell}}\left(L\left(W_{0}(A)\right)\right),
$$

with $L: W_{0}(A) \rightarrow \Lambda(A)$ is the characteristic polynomial map to the undeformed $\Lambda(A)$, with the operations induced from $W_{0}(A)$. 
Lemma 4.9. Let $A=k$ be an algebraically closed field. The multiplication in $\tilde{\mathcal{W}}_{0}^{q}(A)$ obtained as above satisfies

$$
\star: \tilde{\Omega}_{q^{\ell}}\left(L\left(W_{0}(A)\right)\right) \times \tilde{\Omega}_{q^{\ell^{\prime}}}\left(L\left(W_{0}(A)\right)\right) \rightarrow \tilde{\Omega}_{q^{\ell+\ell^{\prime}}}\left(L\left(W_{0}(A)\right)\right) .
$$

Proof. It suffices to check the property on elements of the form $L(E, f)=\prod(1-\alpha t)^{-n(\alpha)}$. With the undeformed product in $\Lambda(A)$ we have

$$
\prod\left(1-\alpha q^{\ell} t\right)^{-n(\alpha)} \star \prod\left(1-\beta q^{\ell^{\prime}} t\right)^{-n(\beta)}=\prod\left(1-\alpha \beta q^{\ell+\ell^{\prime}} t\right)^{-n(\alpha) n(\beta)} .
$$

Proposition 4.10. Let $k$ be an algebraically closed field. The divisor map (3.5) extends to a ring isomorphism $\tilde{\delta}: \tilde{\mathcal{W}}_{0}^{q}(k) \rightarrow \mathbb{Z}[q]\left[k^{*}\right]$ given by

$$
\tilde{\delta}\left(\tilde{\Omega}_{q^{\ell}}(L(E, f))\right)=\delta\left(\prod\left(1-\alpha q^{\ell} t\right)^{-n(\alpha)}\right)=q^{\ell} \sum n(\alpha)[\alpha] .
$$

Combining this with an isomorphism $\sigma: \overline{\mathbb{F}}_{p}^{*} \rightarrow(\mathbb{Q} / \mathbb{Z})^{(p)}$ as in $[5]$ gives an isomorphism

$$
\hat{\sigma}_{q}=\sigma \circ \tilde{\delta}: \tilde{\mathcal{W}}_{0}^{q}\left(\overline{\mathbb{F}}_{p}^{*}\right) \rightarrow \mathbb{Z}[q]\left[(\mathbb{Q} / \mathbb{Z})^{(p)}\right] .
$$

Proof. The argument is exactly as in the previous case and in Proposition 2.3 of [5]. The compatibility with multiplication follows from the previous Lemma.

4.4. $\mathbb{A}^{\ell}$-deformed Bost-Connes endomorphisms. Let $\hat{\sigma}_{q}: \tilde{\mathcal{W}}_{0}^{q}\left(\overline{\mathbb{F}}_{p}^{*}\right) \rightarrow \mathbb{Z}[q]\left[(\mathbb{Q} / \mathbb{Z})^{(p)}\right]$ be as in $(4.19)$, with $\mathbb{Z}[q]\left[(\mathbb{Q} / \mathbb{Z})^{(p)}\right] \subset \mathbb{Z}[q][\mathbb{Q} / \mathbb{Z}]$. We construct endomorphisms $\hat{\sigma}_{n, q}$ of the ring $\mathbb{Z}[q]\left[(\mathbb{Q} / \mathbb{Z})^{(p)}\right]$ that satisfy

$$
\hat{\sigma}_{n, q} \circ \hat{\sigma}_{q}=\hat{\sigma}_{q} \circ F_{n}
$$

with $F_{n}$ the Frobenius on $\tilde{\mathcal{W}}_{0}^{q}\left(\overline{\mathbb{F}}_{p}^{*}\right)$.

Lemma 4.11. Let $E(r, k)=q^{k} e(r) \in \mathbb{Z}[q][\mathbb{Q} / \mathbb{Z}]$, with $e(r)$ the generators of $\mathbb{Z}[\mathbb{Q} / \mathbb{Z}]$. The endomorphisms

$$
\hat{\sigma}_{n, q}(E(r, k))=q^{n k} e(n r)=E(n r, n k)
$$

satisfy the compatibility condition of (4.20).

Proof. It suffices to check that the Frobenius action on $\tilde{\mathcal{W}}_{0}^{q}(k)$ is given by

$$
F_{n} \Omega_{q^{\ell}}(L(E, f))=\Omega_{q^{n \ell}} F_{n} L(E, f)
$$

since we have

$$
F_{n} \prod\left(1-\alpha q^{\ell} t\right)^{-n(\alpha)}=\prod\left(1-\alpha^{n} q^{n \ell} t\right)^{-n(\alpha)}=\Omega_{q^{n \ell}} L\left(E, f^{n}\right) .
$$

The divisor map then gives $\tilde{\delta}\left(F_{n} \Omega_{q^{\ell}}(L(E, f))\right)=q^{n \ell} \sum n(\alpha)\left[\alpha^{n}\right]$. For $k=\overline{\mathbb{F}}_{p}^{*}$, this is the image of $\tilde{\delta}\left(\Omega_{q^{\ell}}(L(E, f))\right)=q^{\ell} \sum n(\alpha)[\alpha]$ under an endomorphism $\hat{\sigma}_{n, q}$ that induces (4.21) on $\mathbb{Z}[\mathbb{Q} / \mathbb{Z}]$. 
The compatibility with the Verschiebung $V_{n}$ is more subtle.

In geometric terms, that is, for elements of the Witt ring that are zeta function of a scheme of finite type over $k$, the Verschiebung corresponds to the Weil restriction of scalars. Namely, if $X$ is a scheme of finite type over an extension $k^{\prime}=\mathbb{F}_{p^{n r}}$ of $k=\mathbb{F}_{p^{r}}$, the Verschiebung acts on the zeta function $V_{n} Z(X, t)=Z\left(R_{n} X, t\right)$, where $R_{n} X=\operatorname{Res}_{k^{\prime} / k} X$ is the scheme over $k$ obtained by restriction of scalars from $k^{\prime}$ to $k$, see [24] for more details.

In our setting, we have the following compatibility condition between Verschiebung and the deformations $\tilde{\Omega}_{q^{\ell}}$.

Lemma 4.12. Let $A=k$ be an algebraically closed field. The Verschiebung $V_{n}$ on $W_{0}(A)$ satisfies

$$
V_{n} \circ \tilde{\Omega}_{q^{n \ell}}=\Omega_{q^{\ell}} \circ V_{n} .
$$

Proof. For $(E, f) \in W_{0}(A)$ with $L(E, f)=\prod(1-\alpha t)^{-n(\alpha)}$ we have

$$
V_{n}(L(E, f))=L\left(V_{n}(E, f)\right)=L\left(\left(E^{\oplus n}, V_{n}(f)\right)\right.
$$

with

$$
V_{n}(f)=\left(\begin{array}{ccccc}
0 & 0 & \ldots & \ldots & f \\
1 & 0 & 0 & \ldots & 0 \\
\ldots & \ldots & \ldots & \ldots & \ldots \\
0 & 0 & 0 & \ldots . & 0
\end{array}\right),
$$

hence $L\left(V_{n}(E, f)\right)=\prod\left(1-\omega_{i, n} t\right)^{-n(\alpha)}$, where $\omega_{i, n}^{n}=\alpha$ for $i=1, \ldots, n$. Thus, we have

$$
\Omega_{q^{\ell}} V_{n}(L(E, f))=\prod\left(1-\omega_{i, n} q^{\ell} t\right)^{-n(\alpha)}=V_{n}\left(\prod\left(1-\alpha q^{n \ell} t\right)^{-n(\alpha)}\right)=V_{n} \Omega_{q^{n \ell}}(L(E, f)),
$$

hence $V_{n}$ satisfies (4.23).

This shows that, in order to extend the Verschiebung $V_{n}$ to the entire $\tilde{\mathcal{W}}_{0}^{q}(A)$, compatibly with its action on $W_{0}(A)$ and with the relation (4.23), one needs to extend the deformation

$$
\tilde{\Omega}_{q^{\ell}}: \prod(1-\alpha t)^{-n(\alpha)} \mapsto \prod\left(1-\alpha q^{\ell} t\right)^{-n(\alpha)}
$$

to include rational powers of $q$, so that $V_{n} \circ \tilde{\Omega}_{q^{\ell}}$ can be defined, compatibly with (4.23), when $n$ does not divide $\ell$. In terms of our geometric interpretation, these $q$-deformations arise from products with affine spaces. Equivalently, in motivic terms, they are given by products with powers of the Lefschetz motive $\mathbb{L}$. Thus, we can frame an appropriate extension of the action of the Verschiebung in terms of the roots of Tate motives discussed in [14].

4.5. Roots of Tate motives. In the geometric setting, the zeta functions $Z(X, t)$ of schemes of finite type over $k=\mathbb{F}_{p^{r}}$ determine a ring homomorphism from the Grothendieck $\operatorname{ring} K_{0}\left(\mathcal{V}_{\mathbb{F}_{p^{r}}}\right)$ of varieties over $\mathbb{F}_{p^{r}}$ to the Witt ring $W(\mathbb{Z})$, see Theorem 2.1 of [24]. The Grothendieck ring $K_{0}\left(\mathcal{V}_{k}\right)$ is generated by isomorphism classes $[X]$ of schemes of finite type over $k$ with relations $[X]=[Y]+[X \backslash Y]$ for a closed subscheme $Y$ of $X$ and $[X] \cdot[Y]=$ $[X \times Y]$. Classes in $K_{0}\left(\mathcal{V}_{k}\right)$ are sometimes referred to as "virtual motives". The Lefschetz motive $\mathbb{L}$ is the class of the affine line $\mathbb{L}=\left[\mathbb{A}^{1}\right]$ in $K_{0}\left(\mathcal{V}_{k}\right)$. The Tate subring $\mathbb{Z}[\mathbb{L}]$ of $K_{0}\left(\mathcal{V}_{k}\right)$ maps to the subring of the Witt ring generated by the zeta functions $Z\left(\mathbb{A}^{\ell}, t\right)=\left(1-q^{\ell} t\right)^{-1}$.

The idea of introducing roots of Tate motives was first suggested in [16]. A geometric construction of a square root $\mathbb{Q}(1 / 2)$ of the Tate motive $\mathbb{Q}(1)$ in terms of supersingular elliptic curves was given in [25]. A categorical construction of a square root of the Tate 
motive was given in $\S 3.4$ of [13] and generalized to arbitrary roots $\mathbb{Q}(r)$, for $r \in \mathbb{Q}_{+}$, in [14]. We recall here briefly this formal categorical definition. Let $\mathcal{T}=\operatorname{Num}_{\mathbb{Q}}(k)$ be the Tannakian category of numerical pure motives over $k$ with motivic Galois group $G=G(\mathcal{T})$. Consider the homomorphisms $\sigma_{n}: \mathbb{G}_{m} \rightarrow \mathbb{G}_{m}$ given by $\sigma_{n}: \lambda \mapsto \lambda^{n}$, and consider the fibered product of $G$ and $\mathbb{G}_{m}$

$$
G^{(n)}=\left\{(g, \lambda) \in G \times \mathbb{G}_{m}: t(g)=\sigma_{n}(\lambda)\right\},
$$

where $t: G \rightarrow \mathbb{G}_{m}$ is the group homomorphism that corresponds to the inclusion of the Tate motives (with Galois group $\mathbb{G}_{m}$ ) inside $\operatorname{Num}_{\mathbb{Q}}(k)$. The group $G^{(n)}$ is in turn the Galois group of a Tannakian category $\mathcal{T}\left(\mathbb{Q}\left(\frac{1}{n}\right)\right)$ which extends the category $\mathcal{T}$ by an $n$-th root $\mathbb{Q}(1 / n)$ of the Tate motive $\mathbb{Q}(1)$. A more precise description of these categories and their properties is given in $\S 4$ of [14], where the construction of the roots $\mathbb{Q}(1 / n)$ is explained in terms of the primary decomposition of $n \in \mathbb{N}$. Let $\tilde{\mathcal{T}}$ be the Tannakian category that corresponds to the projective limit $\tilde{G}$ of the groups $G^{(n)}$, as in [14, that is, the Tannakian category obtained from $\operatorname{Num}_{\mathbb{Q}}(k)$ by adjoining roots of Tate motives of arbitrary order, $\mathbb{Q}(r)$ with $r \in \mathbb{Q}_{+}$.

At the level of the Grothendieck ring $K_{0}\left(\mathcal{V}_{k}\right)\left[\mathbb{L}^{-1}\right]$ with $\mathbb{L}^{-1}$ the class of the Tate motive $\mathbb{Q}(1)$, the roots $\mathbb{Q}(1 / n)$ correspond to additing new generatots $\mathbb{L}^{1 / n}$ and their inverses. The Tate part of the resulting Grothendieck ring $K_{0}(\tilde{\mathcal{T}})$ would then consist of the ring $\mathbb{Z}\left[\mathbb{L}^{r}: r \in \mathbb{Q}_{+}\right]$, since $K_{0}\left(\mathcal{T}\left(\mathbb{Q}\left(\frac{1}{n}\right)\right)\right)=K_{0}(\mathcal{T})[s] /\left(s^{n}-\mathbb{L}\right)$.

Lemma 4.13. The ring homomorphism $Z: K_{0}\left(\mathcal{V}_{k}\right) \rightarrow W(\mathbb{Z})$ given by the zeta functions $Z(X, t)$ extends uniquely to a ring homomorphism $Z: K_{0}(\tilde{\mathcal{T}}) \rightarrow W(K)$, obtained by setting $Z\left(\mathbb{L}^{r}, t\right):=\left(1-q^{r} t\right)^{-1}$ for $r \in \mathbb{Q}_{+}$. Here $K$ is a field that contains $\mathbb{Z}$ and all the non-negative real roots $q^{r}$, for $r \in \mathbb{Q}_{+}$.

Proof. By the description above of the Grothendieck ring $K_{0}\left(\mathcal{T}\left(\mathbb{Q}\left(\frac{1}{n}\right)\right)\right)=K_{0}(\mathcal{T})[s] /\left(s^{n}-\right.$ $\mathbb{L})$, in order to extend the zeta function homomorphism we need to assign to the additional generator $s$ an element $Z(s)$ in the Witt ring with the property that the $n$-fold product $Z(s)^{\star n}=[1]_{w}$, where the unit $[1]_{w}$ in the Witt ring is $[1]_{w}:=(1-t)^{-1}$. Assigning $Z(s, t)=$ $\left(1-q^{1 / n} t\right)^{-1} \in W(K)$ satisfies this requirement.

4.6. Deformed Bost-Connes algebra. We then extend the $\mathbb{A}^{\ell}$-deformation described above, by including roots $\mathbb{L}^{r}, r \in \mathbb{Q}_{+}$of the Lefschetz motive $\mathbb{L}^{r}$, as explained above. This will have the effect of rendering the endomorphisms $\sigma_{n, q}$ invertible in their action on the $q$ variable, while the action on the generators $e(r)$ of $\mathbb{Q} / \mathbb{Z}$ remains unchanged. The following elementary fact explains the main idea.

Lemma 4.14. Consider the endomorphisms $\sigma_{n}: \mathbb{Z}[q] \rightarrow \mathbb{Z}[q]$ determined by $\sigma_{n}: q \mapsto q^{n}$. The direct limit is given by

$$
\mathcal{R}=\underset{n}{\lim }\left(\sigma_{n}: \mathbb{Z}[q] \rightarrow \mathbb{Z}[q]\right)=\mathbb{Z}\left[q^{r}: r \in \mathbb{Q}_{+}\right],
$$

the polynomial ring in the fractional powers $q^{r}$. The induced endomorphism $\sigma_{n}$ on the limit $\mathbb{Z}\left[q^{r}: r \in \mathbb{Q}_{+}\right]$has inverse $\rho_{n}: q^{r} \mapsto q^{r / n}$.

We consider the following modification of the deformed Witt ring $\tilde{\mathcal{W}}_{0}^{q}(A)$. 
Definition 4.15. For $r \in \mathbb{Q}_{+}$. For $A=k$ an algebraically closed field and $(E, f) \in W_{0}(A)$ with $L(E, f)=\prod(1-\alpha t)^{-n(\alpha)}$, let

$$
\tilde{\Omega}_{q^{r}}(L(A, f))=\prod\left(1-\alpha q^{r} t\right)^{-n(\alpha)},
$$

where we treat $q$ and $q^{r}$ as formal variables. We set

$$
\hat{\mathcal{W}}_{0}^{q}(A)=\oplus_{r \in \mathbb{Q}_{+}} \Omega_{q^{r}}\left(L\left(W_{0}(A)\right),\right.
$$

with the operations induced from $W_{0}(A)$.

As in Lemma 4.9, we have

$$
\star: \tilde{\Omega}_{q^{r}}\left(L\left(W_{0}(A)\right)\right) \times \tilde{\Omega}_{q^{r^{\prime}}}\left(L\left(W_{0}(A)\right)\right) \rightarrow \tilde{\Omega}_{q^{\ell+r^{\prime}}}\left(L\left(W_{0}(A)\right)\right) .
$$

Lemma 4.16. The Frobenius and Verschiebung on $W_{0}(A)$ extend to $\hat{\mathcal{W}}_{0}^{q}(A)$ by

$$
F_{n} \circ \tilde{\Omega}_{q^{r}}=\tilde{\Omega}_{q^{n r}} \circ F_{n} \quad \text { and } \quad V_{n} \circ \tilde{\Omega}_{q^{r}}=\tilde{\Omega}_{q^{r / n}} \circ V_{n} .
$$

Proof. The case of Frobenius is as in (4.22) and the argument for the Verschiebung is analogous to Lemma 4.12 .

Lemma 4.17. The divisor map (3.5) induces a ring isomorphism $\hat{\delta}: \hat{\mathcal{W}}_{0}^{q}(k) \rightarrow \mathcal{R}\left[k^{*}\right]$, with the ring $\mathcal{R}$ as in (4.24). Combining this with an isomorphism $\sigma: \overline{\mathbb{F}}_{p}^{*} \rightarrow(\mathbb{Q} / \mathbb{Z})^{(p)}$ as in $[5]$ gives an isomorphism

$$
\hat{\sigma}_{q}=\sigma \circ \hat{\delta}: \hat{\mathcal{W}}_{0}^{q}\left(\overline{\mathbb{F}}_{p}^{*}\right) \rightarrow \mathcal{R}\left[(\mathbb{Q} / \mathbb{Z})^{(p)}\right] .
$$

Proof. The argument is exactly as in Proposition 4.10.

Proposition 4.18. Let $k=\overline{\mathbb{F}}_{p}$. The q-deformed integral Bost-Connes algebra $\mathcal{A}_{\mathbb{Z}, q}$ determined by the deformation (4.26) of the Witt ring $W_{0}(k)$ is generated by the group ring $\mathcal{R}[\mathbb{Q} / \mathbb{Z}]$, with $\mathcal{R}$ as in (4.24) together with elements $\tilde{\mu}_{n}$ and $\mu_{n}^{*}$ satisfying the relations

$$
\begin{aligned}
& \tilde{\mu}_{n} E\left(r, r^{\prime}\right) \mu_{n}^{*}=\hat{\rho}_{n, q}\left(E\left(r, r^{\prime}\right)\right), \\
& \mu_{n}^{*} E\left(r, r^{\prime}\right)=\hat{\sigma}_{n, q}\left(E\left(r, r^{\prime}\right)\right) \mu_{n}^{*} \\
& E\left(r, r^{\prime}\right) \tilde{\mu}_{n}=\tilde{\mu}_{n} \hat{\sigma}_{n, q}\left(E\left(r, r^{\prime}\right)\right),
\end{aligned}
$$

where $E\left(r, r^{\prime}\right)=q^{r} e\left(r^{\prime}\right)$ are the generators of $\mathcal{R}[\mathbb{Q} / \mathbb{Z}]$ and $\hat{\sigma}_{n, q}: \mathcal{R}[\mathbb{Q} / \mathbb{Z}] \rightarrow \mathcal{R}[\mathbb{Q} / \mathbb{Z}]$ are the endomorphisms

$$
\hat{\sigma}_{n, q}\left(E\left(r, r^{\prime}\right)\right)=E\left(n r, n r^{\prime}\right)
$$

The $\hat{\rho}_{n, q}$ are defined by

$$
\hat{\rho}_{n, q}\left(E\left(r, r^{\prime}\right)\right)=\sum_{n s=r^{\prime}} E\left(\frac{r}{n}, s\right)
$$

These satisfy the relations

$$
\begin{array}{r}
\tilde{\mu}_{n m}=\tilde{\mu}_{n} \tilde{\mu}_{m}, \quad m, n \in \mathbb{N}, \quad \mu_{n m}^{*}=\mu_{n}^{*} \mu_{m}^{*}, \quad m, n \in \mathbb{N}, \\
\mu_{n}^{*} \tilde{\mu}_{n}=n, \quad n \in \mathbb{N}, \quad \tilde{\mu}_{n} \mu_{n}^{*}=\mu_{n}^{*} \tilde{\mu}_{n}, \quad(n, m)=1,
\end{array}
$$

as in the original integral Bost-Connes algebra. 
Proof. The endomorphisms $\hat{\sigma}_{n, q}$ are constructed so as to satisfy the compatibility with the Frobenius map $F_{n}$ acting on $\hat{\mathcal{W}}_{0}^{q}(k)$,

$$
\hat{\sigma}_{n, q} \circ \hat{\sigma}_{q}=\hat{\sigma}_{q} \circ F_{n},
$$

with $\hat{\sigma}_{q}$ as in (4.29). This determines $\hat{\sigma}_{n, q}$ to be of the form

$$
\hat{\sigma}_{n, q}\left(E\left(r, r^{\prime}\right)\right)=q^{n r} e\left(n r^{\prime}\right)=E\left(n r, n r^{\prime}\right) .
$$

In turn the $\hat{\rho}_{n, q}$ are constructed so as to be compatible with the Verschiebung $V_{n}$ on $\hat{\mathcal{W}}_{0}^{q}(k)$,

$$
\hat{\rho}_{n, q} \circ \hat{\sigma}_{q}=\hat{\sigma}_{q} \circ V_{n} .
$$

This determines the $\hat{\rho}_{n, q}$ to be given by

$$
\hat{\rho}_{n, q}\left(E\left(r, r^{\prime}\right)\right)=q^{r / n} \sum_{n s=r^{\prime}} e\left(r^{\prime}\right)=\sum_{n s=r^{\prime}} E\left(\frac{r}{n}, s\right) .
$$

Lemma 4.19. Let $\mathcal{R}_{\mathbb{Q}}=\mathcal{R} \otimes_{\mathbb{Z}} \mathbb{Q}=\mathbb{Q}\left[q^{r}: r \in \mathbb{Q}_{+}\right]$. The rational $q$-deformed Bost-Connes algebra $\mathcal{A}_{\mathbb{Q}, q}$ is the semigroup crossed product $\mathcal{R}_{\mathbb{Q}}[\mathbb{Q} / \mathbb{Z}] \rtimes_{\rho} \mathbb{N}$ with generators $E\left(r, r^{\prime}\right)=$ $q^{r} e\left(r^{\prime}\right)$ and $\mu_{n}, \mu_{n}^{*}$ and with the semigroup crossed product action given by

$$
\mu_{n} E\left(r, r^{\prime}\right) \mu_{n}^{*}=\frac{1}{n} \sum_{n s=r^{\prime}} E\left(\frac{r}{n}, s\right)=\rho_{n, q}\left(E\left(r, r^{\prime}\right)\right),
$$

and the relations $E\left(r, r^{\prime}\right) E\left(s, s^{\prime}\right)=E\left(r+s, r^{\prime}+s^{\prime}\right)$ and

$$
\begin{array}{ll}
\mu_{n}^{*} \mu_{n}=1, \forall n, & \mu_{n m}=\mu_{n} \mu_{m}, \forall n, m, \\
\mu_{n}^{*} \mu_{m}^{*}=\mu_{m}^{*} \mu_{n}^{*}, \forall n, m, & \mu_{n} \mu_{m}^{*}=\mu_{m}^{*} \mu_{n}, \text { for }(n, m)=1 .
\end{array}
$$

Proof. This follows directly from the integral algebra $\mathcal{A}_{\mathbb{Z}, q}$ by taking $\rho_{n}=\frac{1}{n} \tilde{\rho}_{n}$. The relation between the rational and the integral algebra is as in the original case, see [7].

4.7. The role of the $q$-integers. Intuitively, one would expect the $q$-deformation of the Bost-Connes algebra to replace the integers $n \in \mathbb{N}$ with the $q$-integers $[n]_{q}=1+q+\cdots+q^{n-1}$. However, it is clear that this cannot be done just directly, since the $q$-integers $[n]_{q}$ do not behave well with respect to the semigroup property that is crucial to the structure of the Bost-Connes algebra.

However, one can see a geometric form of the $q$-integers in the deformed Witt rings $\tilde{\mathcal{W}}_{0}^{q}(A)$ and $\hat{\mathcal{W}}_{0}^{q}(A)$, by comparing it again with the case of the zeta functions of schemes over finite fields. We have seen that mapping $L(E, f)=\prod(1-\alpha t)^{-n(\alpha)}$ to $\tilde{\Omega}_{q^{\ell}}(L(E, f))=\prod(1-$ $\left.\alpha q^{\ell} t\right)^{-n(\alpha)}$ corresponds, in the case of zeta functions, to mapping $Z(X, t)$ to $Z\left(X \times \mathbb{A}^{\ell}, t\right)$. Similarly, we can consider, for a scheme $X$ of finite type over $k$, the transformation that maps $Z(X, t)$ to

$$
Z\left(X \times \mathbb{P}^{n}, t\right)=Z(X, t) \star Z\left(\mathbb{P}^{n}, t\right) .
$$

The zeta function of $\mathbb{P}^{n}$ is given by

$$
Z\left(\mathbb{P}^{n}, t\right)=\prod_{i=0}^{n}\left(1-q^{i} t\right)^{-1}=[1]_{w}+{ }_{w}[q]_{w}+{ }_{w}[q]_{w}^{\star 2}+{ }_{w} \cdots+{ }_{w}[q]_{w}^{\star n},
$$

where we denote by $[a]_{w}:=(1-a t)^{-1}$ in the Witt ring. Thus $Z\left(\mathbb{P}^{n}, t\right)$ corresponds to the $q$-integer $[n+1]_{q}$ where sum and multiplication are replaced by the corresponding 
sum an multiplication in the Witt ring. This simply reflects the decomposition $\mathbb{P}^{n}=$ $\mathbb{A}^{0} \sqcup \mathbb{A}^{1} \sqcup \cdots \sqcup \mathbb{A}^{n}$ and the corresponding counting of points $\# \mathbb{P}^{n}\left(\mathbb{F}_{q}\right)=[n+1]_{q}$. The lack of semigroup structure of the $q$-analogs $[n]_{q}$ corresponds geometrically to the fact that a product of projective spaces $\mathbb{P}^{n} \times \mathbb{P}^{m}$ embeds in a projective space $\mathbb{P}^{(n+1)(m+1)-1}$ via the Segre embedding, but is not itself a projective space. If the zeta function is given by $Z(X, t)=\prod_{r \geq 1}\left(1-t^{r}\right)^{-a_{r}}$, then the map above is given by

$$
Z(X, t) \star[n]_{q}=\prod_{\substack{i=0, \ldots, n-1 \\ r \geq 1}}\left(1-q^{i} t^{r}\right)^{-a_{r}} .
$$

In a similar way, we have an operation in $\tilde{\mathcal{W}}_{0}^{q}(A)$ and $\hat{\mathcal{W}}_{0}^{q}(A)$ : for $L(E, f)=\prod(1-\alpha t)^{-n(\alpha)}$ we have

$$
\Omega_{q^{r}}(L(E, f)) \mapsto \Omega_{q^{r}}(L(E, f)) \star[n]_{q}=\prod_{i=0, \ldots, n-1}\left(1-\alpha q^{r+i} t\right)^{-n(\alpha)} .
$$

4.8. Orbit categories and the Habiro ring. Let $\mathcal{C}$ be an additive category and $F$ a self-equivalence. The orbit category $\mathcal{C} / F$ has objects $\operatorname{Obj}(\mathcal{C} / F)=\operatorname{Obj}(\mathcal{C})$ and morphisms

$$
\operatorname{Hom}_{\mathcal{C} / F}(X, Y):=\oplus_{k \in \mathbb{Z}} \operatorname{Hom}_{\mathcal{C}}\left(X, F^{k}(Y)\right) .
$$

If $\mathcal{C}$ is symmetric monoidal and $F=-\otimes \mathcal{O}$ is given by tensoring with an $\otimes$-invertible object $\mathcal{O}$ in $\mathcal{C}$, the orbit category $\mathcal{C} /-_{\otimes \mathcal{O}}$ is also symmetric monoidal, see $\S 7$ of [28].

Let $\mathcal{T}=\operatorname{Num}_{\mathbb{Q}}(k)$ be the category of numerical motives, as above and let $K_{0}(\mathcal{T})$ be the corresponding Grothendieck ring. Then the Grothendieck ring of the orbit category $\mathcal{T}_{n}:=\mathcal{T} /{ }_{-\otimes \mathbb{Q}(n)}$ can be identified with $K_{0}\left(\mathcal{T}_{n}\right)=K_{0}(\mathcal{T}) /\left(\mathbb{L}^{n}-1\right)$, Proposition 3.6 of [14. While the Grothendieck ring of varieties $K_{0}\left(\mathcal{V}_{k}\right)$ is not the same as the Grothendieck ring of numerical motives, this observation gives an interpretation for the meaning of the quotient rings $K_{0}\left(\mathcal{V}_{k}\right) /\left(\mathbb{L}^{n}-1\right)$. When introducing formal roots of Tate motives, one can similarly consider orbit categories $\mathcal{T}(\mathbb{Q}(1 / n)) /-\otimes \mathbb{Q}(m / n)$ and Grothendieck rings $K_{0}(\mathcal{T}(\mathbb{Q}(1 / n))) /\left(\mathbb{L}^{m / n}-1\right)$. When one restricts to considering only Tate motives with Grothendieck ring $\mathbb{Z}[\mathbb{L}]$, introducing roots of Tate motives and taking orbit categories leads, respectively, to the rings $\mathbb{Z}\left[\mathbb{L}^{r}: r \in \mathbb{Q}_{+}\right]$and the ring

$$
\widehat{\mathbb{Z}[\mathbb{L}}]_{\infty}:={\underset{\iota}{N}}_{\lim _{N}} \mathbb{Z}\left[\mathbb{L}^{r}: r \in \mathbb{Q}_{+}\right] / \mathcal{J}_{N}
$$

where $\mathcal{J}_{N}$ is the ideal generated by the elements $\left(\mathbb{L}^{r}\right)_{N}:=\left(\mathbb{L}^{r N}-1\right) \cdots\left(\mathbb{L}^{r}-1\right)$, see [14]. Equivalently written at the level of counting functions of $\mathbb{F}_{q}$-points

$$
\widehat{\mathbb{Z}[q]}]_{\infty}:={\underset{\lim }{N}}_{N} \mathbb{Z}\left[q^{r}: r \in \mathbb{Q}_{+}\right] / \mathcal{J}_{N} .
$$

As shown in [14] and [17, the ring (4.36) is the same as the direct limit

$$
\widehat{\mathbb{Z}[q]}]_{\infty}=\underset{n}{\lim }\left(\sigma_{n}: \widehat{\mathbb{Z}[q]} \rightarrow \widehat{\mathbb{Z}[q]}\right)
$$

of the morphisms $\sigma_{n}: \widehat{\mathbb{Z}[q]} \rightarrow \widehat{\mathbb{Z}[q]}$ determined by $\sigma_{n}: q \mapsto q^{n}$, where $\widehat{\mathbb{Z}[q]}$ is the Habiro ring

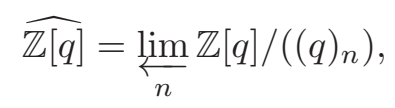

where $(q)_{n}=(1-q)\left(1-q^{2}\right) \cdots\left(1-q^{n}\right)$. 
In terms of the construction of the $q$-deformed Bost-Connes algebra described in Proposition 4.18 and Lemma 4.19, this would correspond to a version of the $q$-deformed algebra where the coefficient ring $\mathcal{R}$ of (4.24) is replaced by $\widehat{\mathcal{R}}:=\widehat{\mathbb{Z}[q]}]_{\infty}$.

Proposition 4.20. The Habiro q-deformed integral Bost-Connes algebra is generated by the group ring $\hat{\mathcal{R}}\left[\mathbb{Q} / \mathbb{Z}\right.$ ] together with elements $\tilde{\mu}_{n}$ and $\mu_{n}^{*}$ satisfying the relations (4.30), (4.31), (4.32). The associated rational algebra is given by the semigroup crossed product $\hat{\mathcal{A}}_{\mathbb{Q}, q}=\hat{\mathcal{R}}[\mathbb{Q} / \mathbb{Z}] \rtimes_{\rho} \mathbb{N}$ with the semigroup action determined by (4.33).

Proof. The compatibility of the operations $\hat{\sigma}_{n, q}$ and $\tilde{\rho}_{n, q}$ with passing to the projective limit $\hat{\mathcal{R}}=\lim _{N} \mathcal{R} / \mathcal{J}_{N}$ can be shown as in Proposition 2.1 and Lemma 2.3 of [17]. The crossed product action in this case remains a semigroup crossed product by $\mathbb{N}$, unlike in the case of the algebra considered in [17] where it becomes a group crossed product by $\mathbb{Q}_{+}^{*}$. Indeed, while the action of $\hat{\sigma}_{n, q}$ on the coefficient ring $\hat{\mathcal{R}}$ is invertible (like it is on $\mathcal{R}$ itself), the action on $\hat{\mathcal{R}}[\mathbb{Q} / \mathbb{Z}]$ still acts like the original Bost-Connes endomorphisms on the generators $e(r)$ of $\mathbb{Z}[\mathbb{Q} / \mathbb{Z}]$, with the partial inverses are given by $\rho_{n, q}$.

4.9. Relation to $\mathbb{F}_{1}$-geometry. The Habiro ring $\widehat{\mathbb{Z}[q]}$ was proposed in $[15$ as a model of analytic functions over $\mathbb{F}_{1}$. The integral Bost-Connes algebra, on the other hand was related to $\mathbb{F}_{1}$-geometry (in the sense of Kapranov-Smirnov [11]) in [7]. In [17] it was shown that a version of the Bost-Connes algebra can be constructed based on the Habiro ring, and in [14] this was related to the orbit categories $\mathcal{T}(\mathbb{Q}(1 / n)) /-\otimes \mathbb{Q}(m / n)$.

The $q$-deformed Bost-Connes algebra of Proposition 4.18 and its variant considered in 4.8 above combine in a natural way the integral Bost-Connes algebra of [7] with the version based on the Habiro ring of [17]. This enriches the algebraic form of $\mathbb{F}_{1}$ and its extensions $\mathbb{F}_{1 m}$ based on roots of unity as in [11], encoded in the integral Bost-Connes algebra, by combining it with the analytic version developed in [15].

4.10. Quantum Statistical Mechanics. Consider first the case where the Bost-Connes algebra itself remains undeformed, as in $\$ 3$. Even on the undeformed Bost-Connes algebra one can consider interesting $q$-deformed time evolutions, that relate the resulting quantum statistical mechanical system to some known $q$-deformations of the Riemann zeta function and polylog functions. The simplest such construction is based on the q-analog of the Riemann zeta function constructed in [26] [27], in the context of a probabilistic approach based on Bernoulli trials with variable probability. In this context, one assumes that the variable $q$ is real with $0 \leq q<1$, as it represents a probability. With this assumption, the $q$-analog of the Riemann zeta function is constructed as follows. For $n \in \mathbb{N}$ with primary decomposition $n=\prod p_{i}^{a_{i}}$, let

$$
v(n)=\sum_{i} a_{i}\left(p_{i}-1\right)
$$

Notice that the function $v(n)$ defined in this way satisfies

$$
v(n m)=v(n)+v(m) .
$$

Also, for $n \in \mathbb{N}$ with primary decomposition $n=\prod p_{i}^{a_{i}}$ we define

$$
\{n\}_{q}=\prod_{i}\left[p_{i}\right]_{q}^{a_{i}}
$$


where $[p]_{q}=1+p+\cdots+p^{n-1}$ is the usual $q$-analog. In other words the $\{n\}_{q}$ are the elements of the multiplicative semigroup $\mathbb{N}_{q}$ generated by the $q$-analogs of the primes. Then one sets

$$
\zeta_{q}(s)=\sum_{n=1}^{\infty} \frac{q^{s \nu(n)}}{\{n\}_{q}^{s}}, \quad \text { with } q<1 .
$$

As shown in [27, this series is convergent for $s>1$ and reduces to the Riemann zeta function when $q \rightarrow 1$. It is also shown in [27] that (4.40) has an Euler product expansion

$$
\zeta_{q}(s)=\prod_{p}\left(1-\frac{q^{s(p-1)}}{[p]_{q}^{s}}\right)^{-1}
$$

In our setting, we regard $q$ as a positive integer, hence $q>1$. The above expression then needs to be modified accordingly. We have

$$
\frac{q^{p-1}}{[p]_{q}}=\frac{q^{p-1}}{1+q+q^{2}+\cdots+q^{p-1}}=\frac{1}{1+q^{-1}+q^{-2}+\cdots+q^{-(p-1)}}=\frac{1}{[p]_{q^{-1}}} .
$$

Thus, by mapping $q \mapsto q^{-1}$, the expression $q^{p-1} /[p]_{q}$ is mapped to $1 /[p]_{q}$ and the version of the zeta function of (4.40) for $q>1$ becomes simply

$$
\zeta_{q}(s)=\sum_{n \geq 1}\{n\}_{q}^{-s}=\prod_{p}\left(1-[p]_{q}^{-s}\right)^{-1}, \quad \text { with } q>1 .
$$

We proceed by defining the time evolution $\sigma_{t, q}$ on the original Bost-Connes algebra $\mathcal{A}_{\mathbb{C}}=\mathcal{A}_{\mathbb{Q}} \otimes_{\mathbb{Q}} \mathbb{C}$ and on its $C^{*}$-algebra completion $\mathcal{A}_{B C}$ is obtained as follows.

Lemma 4.21. Setting $\sigma_{t, q}(e(r))=e(r)$ on the generators $e(r)$ of $\mathbb{C}[\mathbb{Q} / \mathbb{Z}]$ and

$$
\sigma_{t, q}\left(\mu_{n}\right)=\{n\}_{q}^{i t} \mu_{n}
$$

defines a time evolution $\sigma: \mathbb{R} \rightarrow \operatorname{Aut}\left(\mathcal{A}_{\mathbb{C}}\right)$. In the Bost-Connes representations

$$
\pi_{\alpha}: \mathcal{A}_{B C} \rightarrow \mathcal{B}\left(\ell^{2}(\mathbb{N})\right), \quad \text { with } \alpha \in \hat{\mathbb{Z}}^{*},
$$

the time evolution $\sigma_{t, q}$ is generated by the Hamiltonian

$$
H_{q} \epsilon_{n}=\log \{n\}_{q} \epsilon_{n}
$$

and has partition function

$$
Z_{q}(\beta)=\sum_{n=1}^{\infty}\{n\}_{q}^{-\beta}
$$

which is the q-analog zeta function of (4.42).

Proof. The assignment (4.43) defines a time evolution since $\sigma_{t, q}\left(\mu_{n} \mu_{m}\right)=\{n m\}_{q}^{i t} \mu_{n m}=$ $\{n\}_{q}^{i t}\{m\}_{q}^{i t} \mu_{n} \mu_{m}$ and, together with $\sigma_{t, q}(e(r))=e(r)$, is compatible with the relations in the algebra, and clearly $\sigma_{t+s}(a)=\sigma_{t} \sigma_{s}(a)$. In the Bost-Connes representation associated to the choice of an element $\alpha \in \hat{\mathbb{Z}}^{*}$, we have

$$
\begin{gathered}
\mu_{n} \epsilon_{m}=\epsilon_{n m} \\
\pi_{\alpha}(e(r)) \epsilon_{m}=\zeta_{r}^{m} \epsilon_{m}
\end{gathered}
$$

where $\zeta_{r}=\alpha^{*}(e(r))$, identifying $\alpha$ with a choice of embedding of the roots of unity $\mathbb{Q} / \mathbb{Z}$ in $\mathbb{C}$, with $\zeta_{r}$ the image of $e(r)$ under this embedding. The Hamiltonian $H_{q}$ satisfies

$$
e^{i t H_{q}} \pi_{\alpha}(a) e^{-i t H_{q}}=\pi_{\alpha}\left(\sigma_{t, q}(a)\right) .
$$


It suffices to check this condition on $a=\mu_{n}$ acting on basis elements $\epsilon_{m}, e^{i t H_{q}} \mu_{n} e^{-i t H_{q}} \epsilon_{m}=$ $\{m\}_{q}^{-i t}\{n m\}_{q}^{i t} \epsilon_{n m}=\sigma_{t, q}\left(\mu_{n}\right) \epsilon_{m}$. The partition function is then given by

$$
Z_{q}(\beta)=\operatorname{Tr}\left(e^{-\beta H_{q}}\right)=\sum_{n}\left\langle\epsilon_{n}, e^{-\beta H_{q}} \epsilon_{n}\right\rangle=\sum_{n=1}^{\infty}\{n\}_{q}^{-\beta} .
$$

A more interesting case is the construction of quantum statistical mechanical systems associated to the $q$-deformed Bost-Connes algebra of Proposition 4.18 and Lemma 4.19.

Lemma 4.22. The Bost-Connes representations (4.44), (4.47) extend to representations of the deformed algebra $\mathcal{A}_{\mathbb{C}, q}=\mathcal{A}_{\mathbb{Q}, q} \otimes_{\mathbb{Q}} \mathbb{C}$ of Lemma 4.19 on the Hilbert space $\mathcal{H}=\ell^{2}(\mathbb{N}) \otimes$ $\ell^{2}(\Lambda)$ with $\Lambda=q^{\mathbb{Q}_{+}}$, given by

$$
\begin{gathered}
\mu_{n} \epsilon_{m, \lambda}=\epsilon_{n m, \lambda^{1 / n}} \\
\pi_{\alpha}\left(E\left(r, r^{\prime}\right)\right) \epsilon_{m, \lambda}=\zeta_{r^{\prime}}^{m} \epsilon_{m, \lambda \cdot q^{r}} \\
\mu_{n}^{*} \epsilon_{m, \lambda}= \begin{cases}\epsilon_{m / n, \lambda^{n}} & n \mid m \\
0 & n \not \supset m .\end{cases}
\end{gathered}
$$

with $E\left(r, r^{\prime}\right)=q^{r} e\left(r^{\prime}\right)$ and $\zeta_{r}=\alpha(e(r))$ for a given $\alpha \in \hat{\mathbb{Z}}^{*}$, and where $\left\{\epsilon_{m, \lambda}\right\}$ with $m \in \mathbb{N}$ and $\lambda \in \Lambda$ is the standard orthonormal basis of $\mathcal{H}$.

Proof. These are the same kinds of representations considered in [18, in the special case where elements $\lambda \in \Lambda$ always have an $n$-th root in $\Lambda$, so that $\mu_{n}^{*} \mu_{n}$ is the identity and not a projector (see Remark 4.10 and Proposition 4.15 of [18]). First observe that, if we let the generators $q^{r}=E(r, 0)$ act on $\epsilon_{m, \lambda}$ by $E(r, 0): \epsilon_{m, \lambda} \mapsto \epsilon_{m, \lambda \cdot q^{r}}$, then we need the isometries $\mu_{n}$ to act as $\mu_{n}: \epsilon_{m, \lambda} \mapsto \epsilon_{m n, \lambda^{1 / n}}$ (which in turn determines the action of $\mu_{n}^{*}$ as in (4.48)) in order to satisfy the relations

$$
\mu_{n} E(r, 0) \mu_{n}^{*}=\frac{1}{n} \sum_{n s=0} E\left(\frac{r}{n}, s\right)
$$

in $\mathcal{A}_{\mathbb{C}, q}$. Indeed we have, if $n \mid m$

$$
\mu_{n} E(r, 0) \mu_{n}^{*} \epsilon_{m, \lambda}=\mu_{n} E(r, 0) \epsilon_{m / n, \lambda^{n}}=\mu_{n} \epsilon_{m / n, \lambda^{n} \cdot q^{r}}=\epsilon_{m, \lambda \cdot q^{r / n}}=E\left(\frac{r}{n}, 0\right) \epsilon_{m, \lambda},
$$

or zero if $n$ does not divide $m$, which agrees with (4.49). One similary checks compatibility with the other relations:

$$
\mu_{n} E\left(r, r^{\prime}\right) \mu_{n}^{*}=n^{-1} \sum_{n s=r^{\prime}} E\left(\frac{r}{n}, s\right)=\rho_{n}\left(E\left(r, r^{\prime}\right)\right)
$$

and $\mu_{n}^{*} E\left(r, r^{\prime}\right) \mu_{n}=\sigma_{n}\left(E\left(r, r^{\prime}\right)\right)=E\left(n r, n r^{\prime}\right)$, as well as $E\left(r, r^{\prime}\right) E\left(s, s^{\prime}\right)=E\left(r+r^{\prime}, s+s^{\prime}\right)$ and the relations (4.34).

In order to construct time evolutions that have a convergent partition function $\operatorname{Tr}\left(e^{-\beta H}\right)$ for sufficiently large $\beta$, it is convenient to enlarge the algebra by additional "weight operators", as in [18]. We define the resulting algebra as follows. 
Definition 4.23. The extended $q$-Bost-Connes algebra $\mathcal{A}_{\mathbb{Q}, q}^{w}$ is generated by $\mathcal{R}[\mathbb{Q} / \mathbb{Z}]$ and generators $\mu_{n}$ and $\mu_{n}^{*}$ satisfying the relations (4.33), (4.34), and additional generators given by the weight operators $\omega_{z}(\lambda)$ for $\lambda \in \Lambda=q^{\mathbb{Q}_{+}}$and $z \in U(1)$ satisfying the relations

$$
\begin{gathered}
\omega_{z}\left(\lambda_{1} \lambda_{2}\right)=\omega_{z}\left(\lambda_{1}\right) \omega_{z}\left(\lambda_{2}\right), \quad \omega_{z}\left(\lambda^{-1}\right)=\omega_{z}(\lambda)^{-1} \\
\omega_{z}(\lambda) E\left(r, r^{\prime}\right)=E\left(r, r^{\prime}\right) \omega_{z}(\lambda), \quad \omega_{z}(\lambda) \mu_{n}=\mu_{n} \omega_{z}(\lambda)^{n}, \quad \mu_{n}^{*} \omega_{z}(\lambda)=\omega_{z}(\lambda)^{n} \mu_{n}^{*} .
\end{gathered}
$$

Lemma 4.24. Suppose given a group homomorphism $h: \Lambda \rightarrow \mathbb{R}_{+}^{*}$ and an element $\alpha \in \hat{\mathbb{Z}}^{*}$. The representation of Lemma 4.20 mapping $\pi_{\alpha}: \mathcal{A}_{\mathbb{C}, q} \rightarrow \mathcal{B}\left(\ell^{2}(\mathbb{N} \times \Lambda)\right)$, for $\alpha \in \hat{\mathbb{Z}}^{*}$, extends to a representation of $\mathcal{A}_{\mathbb{C}, q}^{w}=\mathcal{A}_{\mathbb{Q}, q}^{w} \otimes_{\mathbb{Q}} \mathbb{C}$ by setting

$$
\omega_{z}(\lambda) \epsilon_{m, \eta}=h(\lambda)^{m z} \epsilon_{m, \eta},
$$

independently of $\alpha \in \hat{\mathbb{Z}}^{*}$, and letting the other generators $E\left(r, r^{\prime}\right), \mu_{n}$ and $\mu_{n}^{*}$ act as in (4.48).

Proof. The argument is exactly as in Proposition 4.15 and Proposition 4.27 of [18, with the only difference that in our case we have $\mu_{n}^{*} \mu_{n}=1$ since elements $\lambda \in \Lambda$ always have an $n$-th $\operatorname{root} \lambda^{1 / n}$ in $\Lambda$.

We can then define a time evolution as in [18] in the following way.

Lemma 4.25. Setting

$$
\sigma_{t}\left(E\left(r, r^{\prime}\right)\right)=\omega_{-i t}\left(q^{r}\right) E\left(r, r^{\prime}\right), \quad \sigma_{t}\left(\mu_{n}\right)=n^{i t} \mu_{n}, \quad \sigma_{t}\left(\omega_{z}(\lambda)\right)=\omega_{z}(\lambda)
$$

defines a time evolution on $\mathcal{A}_{\mathbb{C}, q}^{w}$. In the representations of Lemma 4.24 this time evolution is generated by the Hamiltonian

$$
H \epsilon_{m, \lambda}=(\log (m)-m \log (h(\lambda))) \epsilon_{m, n} .
$$

Proof. We check that (4.52) determines a time evolution $\sigma: \mathbb{R} \rightarrow \operatorname{Aut}\left(\mathcal{A}_{\mathbb{C}, q}^{w}\right)$ as in Lemma 4.23 of [18]. We have

$$
\begin{gathered}
e^{i t H} \pi_{\alpha}\left(E\left(r, r^{\prime}\right)\right) e^{-i t H} \epsilon_{m, \lambda}=e^{i t H} \pi_{\alpha}\left(E\left(r, r^{\prime}\right)\right) m^{-i t} h(\lambda)^{m i t} \epsilon_{m, \lambda} \\
=e^{i t H} m^{-i t} h(\lambda)^{m i t} \zeta_{r^{\prime}}^{m} \epsilon_{m, \lambda q^{r}}=m^{i t} h\left(\lambda q^{r}\right)^{-m i t} m^{-i t} h(\lambda)^{m i t} \zeta_{r^{\prime}}^{m} \epsilon_{m, \lambda q^{r}}= \\
h\left(q^{r}\right)^{-m i t} \zeta_{r^{\prime}}^{m} \epsilon_{m, \lambda q^{r}}=\omega_{-i t}\left(h\left(q^{r}\right)\right) E\left(r, r^{\prime}\right) \epsilon_{m, \lambda}=\sigma_{t}\left(E\left(r, r^{\prime}\right)\right) \epsilon_{m, \lambda} ; \\
e^{i t H} \mu_{n} e^{-i t H} \epsilon_{m, \lambda}=e^{i t H} \mu_{n} m^{-i t} h(\lambda)^{m i t} \epsilon_{m, \lambda}=e^{i t H} m^{-i t} h(\lambda)^{m i t} \epsilon_{m n, \lambda^{1 / n}} \\
=(m n)^{i t} h\left(\lambda^{1 / n}\right)^{-m n i t} m^{-i t} h(\lambda)^{m i t} \epsilon_{m n, \lambda^{1 / n}}=n^{i t} \mu_{n} \epsilon_{m, \lambda} ; \\
e^{i t H} \mu_{n}^{*} e^{-i t H} \epsilon_{m, \lambda}=e^{i t H} \mu_{n}^{*} m^{-i t} h(\lambda)^{m i t} \epsilon_{m, \lambda}=e^{i t H} m^{-i t} h(\lambda)^{m i t} \epsilon_{m / n, \lambda^{n}} \\
=(m / n)^{i t} h\left(\lambda^{n}\right)^{i t m / n} m^{-i t} h(\lambda)^{m i t} \epsilon_{m / n, \lambda^{n}}=n^{-i t} \mu_{n}^{*} \epsilon_{m, \lambda} ; \\
e^{i t H} \omega_{z}(\eta) e^{-i t H} \epsilon_{m, \lambda}=m^{i t} h(\lambda)^{-m i t} h(\eta)^{z m} m^{-i t} h(\lambda)^{m i t} \epsilon_{m, \lambda}=\omega_{z}(\eta) \epsilon_{m, \lambda} .
\end{gathered}
$$

This shows that the operator $H$ of (4.53) generates the time evolution (4.52) in the representation of Lemma 4.24. 
The choice of the homomorphism $h: \Lambda \rightarrow \mathbb{R}_{+}^{*}$ in the construction of the time evolution (4.52) can then be used to determine the convergence properties of the partition function of the quantum statistical mechanical system $\left(\mathcal{A}_{\mathbb{C}, q}^{w}, \sigma_{t}\right)$, as in [18. We adapt the representations described above as in $\S 4.4$ of [18], by decomposing $\Lambda$ into a countable union of geometric progressions and acting on a Hilbert space that is a tensor product of the $\ell^{2}$-spaces of these countable subsets.

Proposition 4.26. Let $\Lambda=q^{\mathbb{Q}_{+}}$and let $\lambda_{k}=q^{1 / k} \in \Lambda$. Consider the homomorphism $h: \Lambda \rightarrow \mathbb{R}_{+}^{*}$ determined by $h(1)=1$ and $h\left(\lambda_{k}\right)=\left[p_{k}\right]_{q}$ where $p_{k}$ is the $k$-th prime number. Consider a representation $\pi_{\alpha}$ of $\mathcal{A}_{\mathbb{C}, q}^{w}$ on the Hilbert space

$$
\mathcal{H}=\ell^{2}(\mathbb{N}) \otimes \bigotimes_{k} \ell^{2}\left(\lambda_{k}^{\mathbb{Z}_{+}}\right)
$$

satisfying (4.48) and (4.51). Then the time evolution (4.52) is implemented in this representation by the Hamiltonian

$$
H \epsilon_{m, \lambda_{k}^{\ell}}=\left(\log (m)-m \ell \log \left(\left[p_{k}\right]_{q}\right)\right) \epsilon_{m, \lambda_{k}^{\ell}} .
$$

The partition function is given by

$$
Z_{q}(\beta)=\sum_{n \geq 1} \zeta_{q}(n \beta) n^{-\beta}
$$

where $\zeta_{q}(s)$ is the q-analog zeta function of (4.42). The series (4.54) converges for $\beta>3 / 2$.

Proof. As in Lemma 4.25 we have

$$
H \epsilon_{m, \lambda_{k}^{\ell}}=\left(\log (m)-m \ell \log \left(h\left(\lambda_{k}\right)\right)\right) \epsilon_{m, \lambda_{k}^{\ell}}=\left(\log (m)-m \ell \log \left(\left[p_{k}\right]_{q}\right)\right) \epsilon_{m, \lambda_{k}^{\ell}} .
$$

Thus, the partition function gives

$$
\begin{aligned}
& Z_{q}(\beta)=\operatorname{Tr}\left(e^{-\beta H}\right)=\sum_{n \geq 1} \prod_{k} \sum_{\ell \geq 0}\left[p_{k}\right]^{-\ell n \beta} n^{-\beta} \\
& =\sum_{n} n^{-\beta} \prod_{p}\left(1-[p]^{-n \beta}\right)^{-1}=\sum_{n} n^{-\beta} \zeta_{q}(n \beta) .
\end{aligned}
$$

Since $q>1$, we have $[p]_{q}=1+q+\cdots+q^{p-1} \geq p$ and $\{n\}_{q} \geq n$, hence $\zeta_{q}(n \beta) \leq \zeta(n \beta)$, with $\zeta(s)$ the Riemann zeta function, so that the convergence of $Z_{q}(\beta)$ is controlled by the convergence of $\sum_{n} n^{-\beta} \zeta(n \beta)$. The convergence of this series for $\beta>3 / 2$ was shown in Theorem 4.30 of [18].

4.11. Weil numbers as a $q$-deformed Bost-Connes system. Finally, we want to mention another related way of constructing a $q$-deformed Bost-Connes system, which is closely related to the one discussed above. In the approach developed in [18], the data of the BostConnes system, consisting of the group ring $\mathbb{Q}[\mathbb{Q} / \mathbb{Z}]$ with the endomorphisms $\sigma_{n}$ and partial inverses $\rho_{n}$, and the associated algebra $\mathcal{A}_{\mathbb{Q}}=\mathbb{Q}[\mathbb{Q} / \mathbb{Z}] \rtimes_{\rho} \mathbb{N}$, can be generalized to other systems based on data $\left(\Sigma, \sigma_{n}\right)$ endowed with a Galois action, such that the $\sigma_{n}$ and partial inverses $\rho_{n}$ arise from Frobenius and Verschiebung functors on a categorification $\operatorname{Vect}_{\Sigma}{ }_{\Sigma}^{\bar{k}}(k)$ of pairs $\left(V, \oplus_{s \in \Sigma} \bar{V}^{s}\right)$ of a finite dimensional $k$-vector space $V$ and a $\Sigma$-grading of $\bar{V}=V \otimes \bar{k}$. The category $\operatorname{Vect}_{\Sigma}^{\bar{k}}(k)$ is neutral Tannakian with Galois group given by the affine $k$-scheme $\operatorname{Spec}\left(\bar{k}[\Sigma]^{G}\right)$, with the group operation induced from the Hopf structure on $\bar{k}[\Sigma]^{G}$. Associated to such data, endowed with a suitable set of $G$-equivariant embeddings of $\Sigma$ to $\overline{\mathbb{Q}}^{*}$, it 
is possible to construct an algebra $\mathcal{A}_{\left(\Sigma, \sigma_{n}\right)}$ together with a time evolution $\sigma_{t}$, so that the properties of the resulting quantum statistical mechanical system generalize the original properties of the Bost-Connes system. In particular, as shown in Example 4 of $\S 5$ of [18], one such generalization of the Bost-Connes algebra can be constructed for the data $\left(\Sigma, \sigma_{n}\right)$ where $\Sigma=\mathcal{W}(q)$ is the set of Weil numbers, namely the subgroup of $\overline{\mathbb{Q}}^{*}$ given by algebraic numbers $\pi$ such that,

- for every embedding $\rho: \mathbb{Q}[\pi] \rightarrow \mathbb{C}$ one has $|\rho(\pi)|=q^{m / 2}$ for some $m \in \mathbb{Z}$, the weight $m=w(\pi)$

- there is some integer $s$ for which $q^{s} \pi$ is an algebraic integer.

Under the identification $\mathcal{W}(q) \simeq \mathcal{W}_{0}(q) \times \mathbb{Z}$ by $\pi \mapsto\left(\frac{\pi}{\rho \rho(\pi)}, w(\pi)\right)$ he surjective group homomorphisms $\sigma_{n}: \mathcal{W}(q) \rightarrow \mathcal{W}(q)$ are given by $(\pi, m) \mapsto\left(\pi^{n}, n m\right)$. Since roots of unity $\mathbb{Q} / \mathbb{Z}$ are contained inside $\mathcal{W}(q)$ as the subgroup $\mathbb{Q} / \mathbb{Z} \times\{0\} \subset \mathcal{W}_{0}(q) \times \mathbb{Z}$, one can regard the resulting Bost-Connes algebra $\mathcal{A}_{\left(\mathcal{W}(q), \sigma_{n}\right)}$ associated to the datum $\left(\mathcal{W}(q), \sigma_{n}\right)$, as constructed in [18] as another form of $q$-deformation of the original Bost-Connes algebra $\mathcal{A}_{\mathbb{Q}}=\mathcal{A}_{\left(\mathbb{Q} / \mathbb{Z}, \sigma_{n}\right)}$. In terms of zeta functions, Weil numbers correspond to Frobenius eigenvalues of motives over finite fields, [19, hence this can be regarded as another way of using zeta functions as a model for $q$-deforming the Bost-Connes algebra. We refer the readers to [18] for more details.

Acknowledgments. The first author was partially supported by NSF grants DMS-1201512 and PHY-1205440. The second author was supported by a Summer Undergraduate Research Fellowship at Caltech.

\section{REFERENCES}

[1] G. Almkvist, Endomorphisms of finitely generated projective modules over a commutative ring, Ark.Mat. 11 (1973), 263-301.

[2] J.B. Bost, A. Connes, Hecke algebras, Type III factors and phase transitions with spontaneous symmetry breaking in Number Theory, Selecta Math. (New Ser.) Vol.1 (1995) N.3, 411-457.

[3] L. Carlitz, q-Bernoulli numbers and polynomials, Duke Math. J. 15, (1948). 987-1000.

[4] I. Cherednik, On q-analogues of Riemann's zeta function, Selecta Math. (N.S.) 7 (2001), no. 4, $447-491$.

[5] A. Connes, C. Consani, On the arithmetic of the BC-system, J. Noncommut. Geom. 8 (2014) N.3, 873-945.

[6] A. Connes, C. Consani, M. Marcolli, Noncommutative geometry and motives: the thermodynamics of endomotives, Advances in Mathematics, Vol.214 (2007) N.2, 761-831.

[7] A. Connes, C. Consani, M. Marcolli, Fun with $\mathbb{F}_{1}$, Journal of Number Theory, Vol.129 (2009), N.6, $1532-1561$.

[8] A. Connes, M. Marcolli, Noncommutative Geometry, Quantum Fields, and Motives, Colloquium Series, Vol.55, American Mathematical Society, 2008.

[9] K. Habiro, Cyclotomic completions of polynomial rings, Publ. Res. Inst. Math. Sci. 40 (2004), no. 4, 1127-1146.

[10] M. Kaneko, N. Kurokawa and M. Wakayama, A variation of Euler's approach to values of the Riemann zeta function, Kyushu J. Math. 57 (2003), 175-192.

[11] M. Kapranov, A. Smirnov, Cohomology determinants and reciprocity laws: number field case, unpublished manuscript.

[12] K. Kawagoe, M. Wakayama, Y. Yamasaki, q-Analogues of the Riemann zeta, the Dirichlet L-functions, and a crystal zeta function, Forum Math. 20 (2008), no. 1, 1-26.

[13] M. Kontsevich, Y. Soibelman, Cohomological Hall algebra, exponential Hodge structures and motivic Donaldson-Thomas invariants, Commun. Number Theory Phys. 5 (2011), no. 2, 231-352.

[14] C.W.K. Lo, M. Marcolli, $F_{\zeta}$-geometry, Tate motives, and the Habiro ring, Int. J. Number Theory, Vol.11 (2015) N.2, 311-339. 
[15] Yu.I. Manin, Cyclotomy and analytic geometry over $\mathbb{F}_{1}$, in "Quanta of maths", pp. 385-408, Clay Math. Proc., 11, Amer. Math. Soc., 2010.

[16] Yu.I. Manin, Lectures on zeta functions and motives (according to Deninger and Kurokawa), Astérisque, Vol.228 (1995) N.4, 121-163.

[17] M. Marcolli, Cyclotomy and endomotives, P-Adic Numbers, Ultrametric Analysis and Applications, Vol.1 (2009) N.3, 217-263.

[18] M. Marcolli, G. Tabuada, Bost-Connes systems, Categorification, Quantum statistical mechanics, and Weil numbers, Journal of Noncommutative Geometry, 11 (2017), no. 1, 1-49.

[19] J.S. Milne, Motives over finite fields, in "Motives (Seattle, WA, 1991)", 401-459, Proc. Sympos. Pure Math., Vol.55, Part 1, Amer. Math. Soc., 1994.

[20] Y.T. Oh, q-analog of the Möbis function and the cyclotomic identity associated to a profinite group, Adv. in Math. Vol.219 (2008) 852-893.

[21] Y.T. Oh, q-deformed necklace rings and q-Möbius function, J. Algebra, Vol.320 (2008) 1599-1625

[22] Y.T. Oh, Nested Witt vectors and their q-deformations, J. Algebra, Vol.309 (2007) 683-710.

[23] Y.T. Oh, Classification of the ring of Witt vectors and the necklace ring associated with the formal group law $X+Y-q X Y$, Journal of Algebra, Vol.310 (2007) 325-250.

[24] N. Ramachandran, Zeta functions, Grothendieck groups, and the Witt ring, Bull. Sci. Math. Vol.139 (2015) 599-627

[25] N. Ramachandran, Values of zeta functions at $s=1 / 2$, Int. Math. Res. Not. 2005, no. 25, $1519-1541$.

[26] D. Rawlings, A probabilistic approach to some of Euler's number theoretic identities, Trans. Amer. Math. Soc. Vol. 350 (1998) 2939-2951.

[27] D. Rawlings, Bernoulli trials and number theory, Amer. Math. Monthly 101 (1994), no. 10, 948-952.

[28] G. Tabuada, Chow motives versus non-commutative motives, J. Noncommut. Geom. 7 (2013), no. 3, 767-786.

[29] J. Zhao, Multiple q-zeta functions and multiple q-polylogarithms, Ramanujan J. 14 (2007), no. 2, 189221.

Department of Mathematics, California Institute of Technology, 1200 E California Blvd, Pasadena, CA 91125, USA

E-mail address: matilde@caltech.edu

E-mail address: zren@caltech.edu 